\title{
Stress-associated erythropoiesis initiation is regulated by type 1 conventional dendritic cells
}

\author{
Taeg S. Kim, ${ }^{1,2}$ Mark Hanak, ${ }^{1}$ Paul C. Trampont, ${ }^{3}$ and Thomas J. Braciale ${ }^{1,2,4}$ \\ ${ }^{1}$ Beirne B. Carter Center for Immunology Research, ${ }^{2}$ Department of Pathology, ${ }^{3}$ Department of Medicine, Division of Hematology and Oncology, and ${ }^{4}$ Department of Microbiology, University of Virginia, \\ Charlottesville, Virginia, USA.
}

\begin{abstract}
Erythropoiesis is an important response to certain types of stress, including hypoxia, hemorrhage, bone marrow suppression, and anemia, that result in inadequate tissue oxygenation. This stress-induced erythropoiesis is distinct from basal red blood cell generation; however, neither the cellular nor the molecular factors that regulate this process are fully understood. Here, we report that type 1 conventional dendritic cells (cDC1s), which are defined by expression of CD8 $\alpha$ in the mouse and XCR1 and CLEC9 in humans, are critical for induction of erythropoiesis in response to stress. Specifically, using murine models, we determined that engagement of a stress sensor, CD24, on CDC1s upregulates expression of the Kit ligand stem cell factor on these cells. The increased expression of stem cell factor resulted in Kit-mediated proliferative expansion of early erythroid progenitors and, ultimately, transient reticulocytosis in the circulation. Moreover, this stress response was triggered in part by alarmin recognition and was blunted in CD24 sensorand CD8 $\alpha^{+}$DC-deficient animals. The contribution of the CDC1 subset to the initiation of stress erythropoiesis was distinct from the well-recognized role of macrophages in supporting late erythroid maturation. Together, these findings offer insight into the mechanism of stress erythropoiesis and into disorders of erythrocyte generation associated with stress.
\end{abstract}

\section{Introduction}

Erythropoiesis is the process of production of new rbc (also known as erythrocytes). In humans, approximately 2.4 million new erythrocytes are produced per second, normally within the BM (1-3). Within the circulation, the end products of erythropoiesis are represented by mature rbc preceded by enucleated RNAcontaining immediate rbc precursors, the reticulocytes. After entering the circulation, mature rbc typically turn over within 120 days. Erythropoiesis is therefore a dynamic process balancing rbc generation and clearance with the need to maintain sufficient tissue oxygenation.

Under homeostatic or steady-state conditions, the stable generation of new rbc is orchestrated by the concerted action of certain growth factors, notably erythropoietin (EPO) and the Kit ligand stem cell factor (SCF) (4-6) as well as the response of several cell types, particularly erythroid progenitors, at various states of differentiation responding to these growth factors through measured (controlled) proliferation, and cells of the monocyte/macrophage lineage (7).

In contrast to basal erythropoiesis, under conditions of inadequate tissue oxygenation resulting from sustained hypoxia or anemia caused by hemorrhage or BM suppression, e.g., chemotherapy, $\mathrm{BM}$ transplantation, infection, etc., there is a rapid response of the erythropoietic machinery to restore tissue oxygenation - a process termed stress erythropoiesis $(8,9)$. Stress erythropoiesis results in the rapid production of new rbc and is characterized by the rapid expansion of the erythroblast progenitor pool and marked transient eleva-

Conflict of interest: The authors have declared that no conflict of interest exists Submitted: March 13, 2015; Accepted: August 13, 2015.

Reference information: / Clin Invest. 2015;125(10):3965-3980. doi:10.1172/JCI81919. tion of reticulocyte numbers in the circulation $(6,10)$. In humans, the stress erythropoiesis response is believed to be localized primarily to the BM, with rbc generation in sites such as the spleen, with associated splenomegaly only observed when stress erythropoiesis is very prominent. In contrast, in the mouse, stress erythropoiesis is primarily localized to extramedullary sites such as the spleen (where this process results in splenomegaly) and liver (typically in younger animals) and to a lesser extent in the BM (10-12). While stress erythropoiesis is generally considered to be an exaggerated form of basal erythropoiesis and therefore dependent on the aforementioned growth factors, erythroblast progenitors and monocyte/macrophage lineage cells, regulators such as bone morphogenetic protein 4 (BMP4) and Hedgehog signaling, and the availability of self-renewing stress erythrocyte progenitors in these extramedullary sites have also been implicated in the control of this process $(6,10,13)$.

Among the cell types within the mononuclear phagocyte system (MPS), resident (tissue) macrophages at sites of erythropoiesis $(1,2)$ and, in particular, a specific subset of CD169+ macrophages have been implicated as important in supporting the late-stage development and differentiation of erythroid progenitors into rbc, likely through their capacity to provide soluble factors and recycled iron from senescent $\mathrm{rbc}$ and through the elimination of nuclei extruded from maturing end-stage erythroid progenitors $(1,3,14)$.

In this report, we define and characterize a previously unappreciated role for the mononuclear phagocytic cell lineage DCs in regulating the initiation of stress erythropoiesis through engagement of a stress sensor, CD24, displayed at a high level on a specific subset of DCs, i.e., type 1 conventional DCs (cDC1s) (CD8 $\alpha^{+}$ in the mouse, ref. 15; and XCR1 $1^{+} \mathrm{CLEC}^{+}$in the human, refs. 16, 17). Engagement of CD24 in vivo results in rapid early erythroid 
A

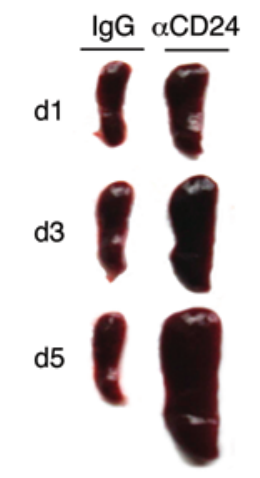

D
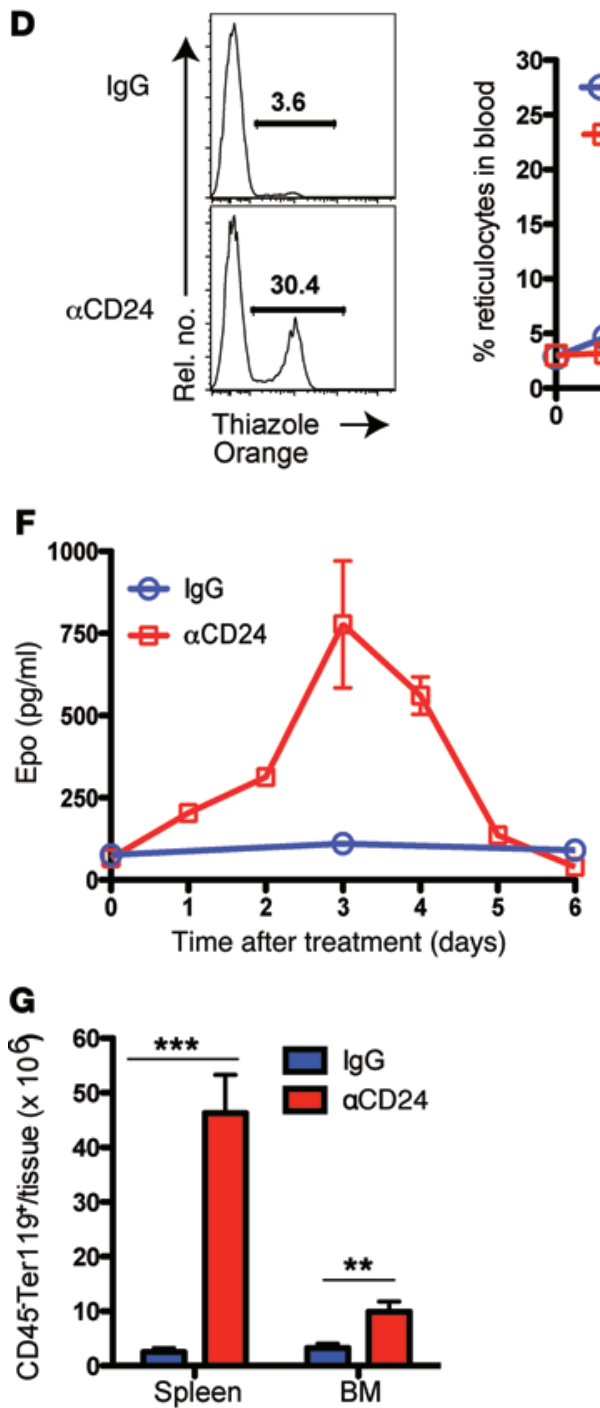

B
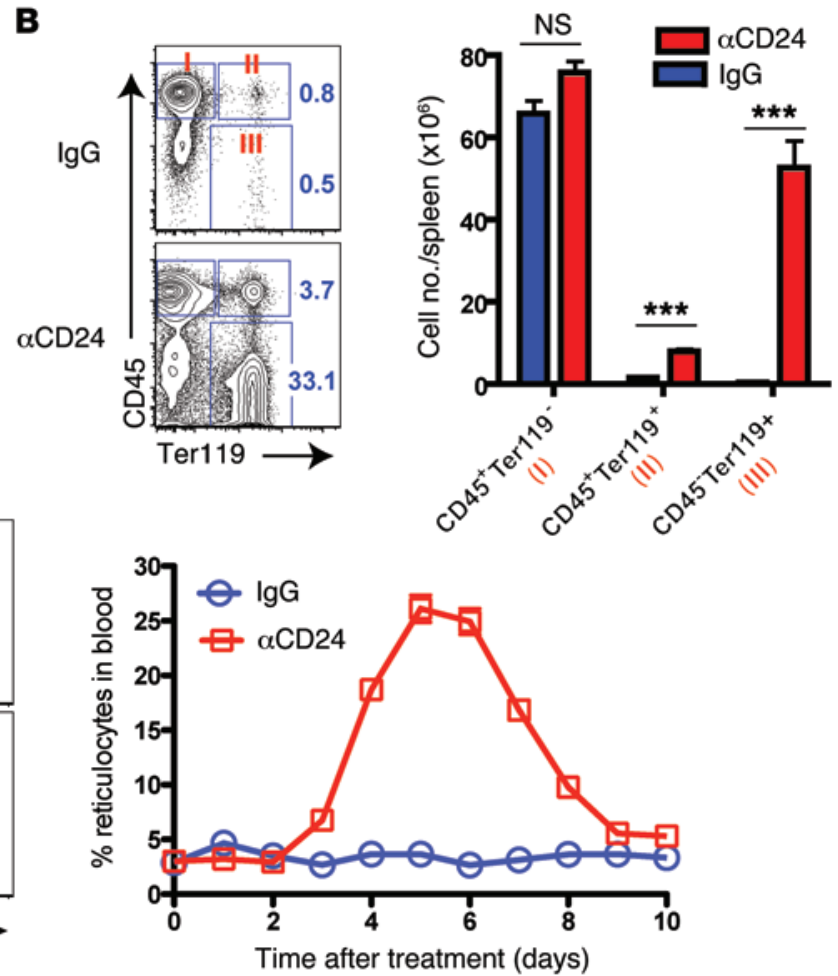

H
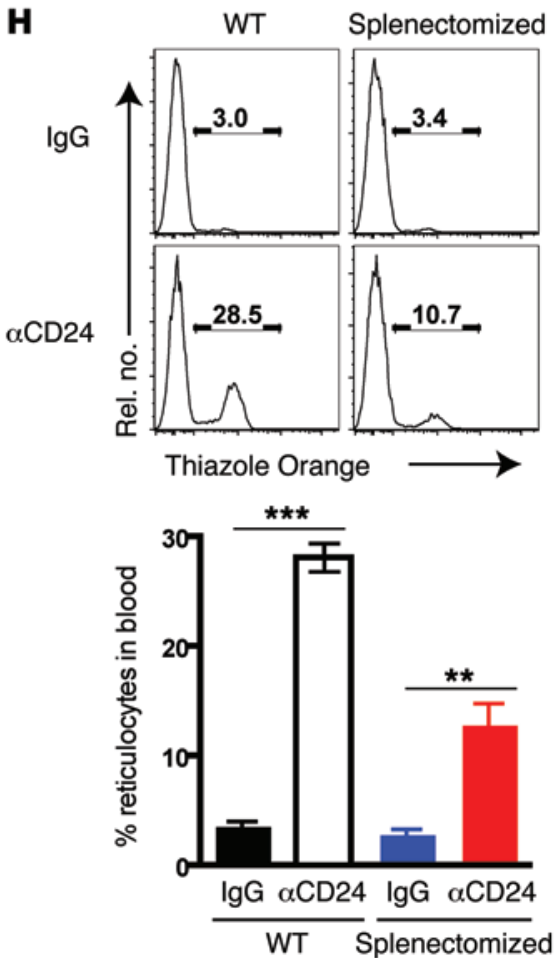

C

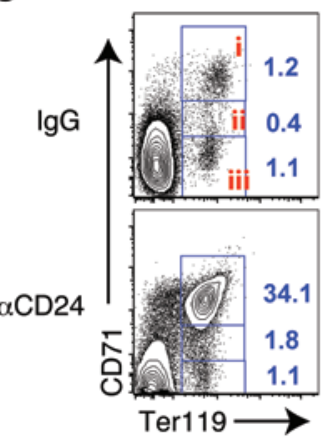

E
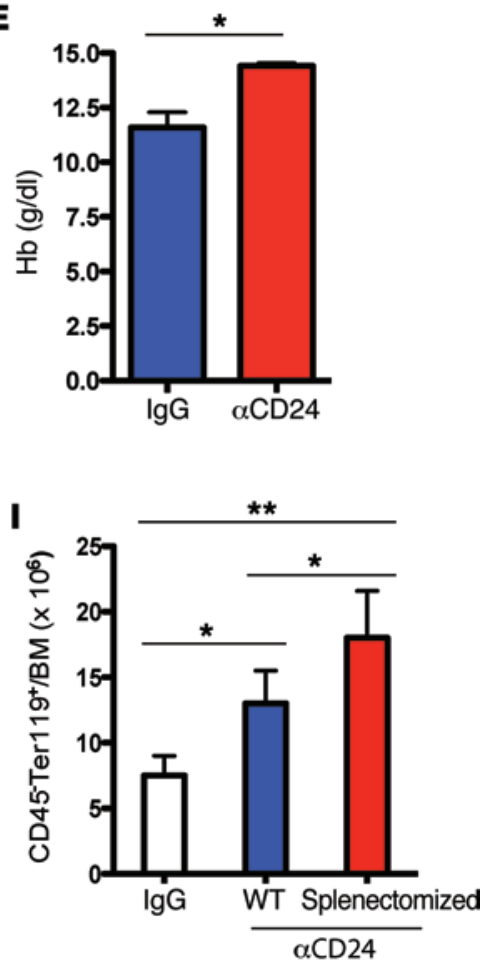

Figure 1. Administration of $\alpha \mathrm{CD} 24$ mAbs induces extramedullary stress erythropoiesis in mice. Mice were infused i.p. with $100 \mu \mathrm{g}$ control rat lgG2b or $\alpha$ CD24 mAbs (clone M1/69) and necropsied on days 1, 3, or 5 after Ab treatment. (A) Representative macroscopic appearance of spleens over time after M1/69 infusion in WT B6 mice $(n>50)$. (B) The rbc-lysed single-cell suspensions prepared from spleens at day 5 after Ab treatment were analyzed for cell types by flow cytometry (left panels) and enumerated (right panels, $n \geq 10$ ): leukocyte (CD45 ${ }^{+}$Ter119', gate i); hemophagocytes (CD45 ${ }^{+}$Ter119 ${ }^{+}$, gate ii), and erythroid progenitor cells (CD45-Ter119+, gate iii). (C and D) Analyses of erythroblastic subsets (C) consisting of basophilic (Ter119+CD71 ${ }^{\text {hi }}$, gate i, least mature), polychromatophilic (Ter119+CD71 med, gate ii), and orthochromatic (Ter119+CD71'10, gate iii) erythroblasts in spleen at day 5 after treatment and reticulocytes in peripheral blood over time $(n>7, \mathbf{D})$. (E) After 2 repeated injections of M1/69 at day 0 and day 6 in Rag1 $1^{-1-}$ mice, sera were collected and measured for Hb levels at day $8(n=4)$. (F) Mice were bled at the indicated days after Ab treatment and measured for EPO levels in the sera $(n=4-6)$.

(C) Total erythroid progenitors per spleen and BM at day 5 are depicted $(n=5)$. (H and I) WT and splenectomized mice were treated i.p. with control Ig or M1/69 and examined for reticulocytes in the blood $(\mathbf{H})$ and erythroid progenitors in the BM $(\mathbf{I})$ at day 5 after Ab treatment $(n=4-6)$. Data represent mean \pm SEM. ${ }^{*} P<0.05$; ${ }^{* *} P<0.01 ;{ }^{* *} P<0.001$ (2-tailed, unpaired Student's $t$ test). Data are pooled from at least 2 independent experiments. 
progenitor expansion and associated robust transient reticulocytosis. Animals deficient in cDC1s exhibit an impaired erythropoietic response to hypoxemia induced by stresses including hypoxia, acute hemorrhage, and BM suppression. Engagement of CD24 on the $\mathrm{CD} 8 \alpha^{+} / \mathrm{XCR}^{+} \mathrm{CLEC} 9^{+}$DCs upregulates the expression of SCF and subsequent Kit-dependent proliferation of early erythroid progenitors. In parallel, CD24 engagement on $\mathrm{CD} 11 \mathrm{c}^{+}$cells stimulates EPO release, resulting in Kit/EPO-mediated reticulocytosis. These and related findings indicate a role for DCs operating prior to but in conjunction with macrophages and EPO in regulating an important response of the body to injury, that is, stress erythropoiesis.

\section{Results}

CD24 engagement in vivo triggers extramedullary (stress-type) erythropoiesis. During an analysis of the contribution of costimulation by $\mathrm{CD} 24$ to the induction of adaptive immune $\mathrm{CD}^{+} \mathrm{T}$ cell responses (18), we observed that parenteral administration of a $\alpha C D 24$ mAbs to naive mice resulted in the rapid, transient development of splenomegaly (Figure 1A). Further analysis of the cellular composition of the enlarged spleens from $\alpha \mathrm{CD} 24 \mathrm{Ab}$-treated animals revealed that the increase in organ size was primarily due to the substantial expansion in the number of Ter119+ erythroid progenitor cells (i.e., erythroblasts) (Figure 1B), in particular, an increase in frequency among cells of the splenic CD71 ${ }^{\text {hi }}$ Ter $119^{+}$erythroblast series (i.e., cells with the expression markers characteristic of erythroblasts with basophilic, polychromatophilic, and orthochromatic morphology) (ref. 19 and Figure 1C). Of note, we also observed increased CD45-Ter119- cells, which include stromal cells and Ter119- erythroid precursors (Figure 1C). To further demonstrate that this response reflected the expansion of erythroid progenitors in the spleen, the activity of burst-forming unit-erythroid (BFU-E) and CFU-erythroid (CFU-E) was measured. We found that BFUE/CFU-E colony-forming activity was readily detectable at days 1 to 2 after $\alpha \mathrm{CD} 24$ treatment, peaking at days 3 to 5 (Supplemental Figure 1A; supplemental material available online with this article; doi:10.1172/JCI81919DS1). In addition, murine erythroid progenitors can be defined by the expression of several surface markers, i.e., Kit ${ }^{+}$Ter119- cells coexpressing CD105 (endoglin). To directly identify erythroid progenitors, splenocytes were examined for Kit and CD105 expression by flow cytometry after $\alpha \mathrm{CD} 24 \mathrm{Ab}$ treatment. We found an expansion of cells expressing CD105 along with Kit (Supplemental Figure 1B). This expansion of erythroid precursors following parenteral $\alpha \mathrm{CD} 24 \mathrm{Ab}$ administration was accompanied by a transient reticulocytosis (Figure 1D and Supplemental Figure $1 \mathrm{C}$ ) and a rise of hemoglobin ( $\mathrm{Hb}$ ) by day 8 after $\mathrm{mAb}$ administration (Figure 1E), which was preceded by a transient rise in serum EPO (Figure $1 \mathrm{~F}$ ). This transient rise in serum EPO was not due to Ab-induced acute anemia, i.e., loss and/or sequestration of erythrocytes, since blood rbc numbers, $\mathrm{Hb}$, and hematocrit (HCT) remained stable at days 1 through 3 following $\mathrm{mAb}$ administration, that is, preceding the maximum increase in serum EPO level (Supplemental Figure 1D). F(ab) ${ }_{2}$ fragments of this $\alpha \mathrm{CD} 24 \mathrm{mAb}$ stimulated erythropoiesis to a corresponding extent and with a tempo identical to that of the administration of the intact mAbs (data not shown). It is also noteworthy that a similar but less dramatic expansion of erythroblasts was likewise observed in the $\mathrm{BM}$ of mice following parenteral $\alpha \mathrm{CD} 24 \mathrm{mAb}$ administration (Figure 1G). Treatment of mice with isotype control Abs had no effect on these parameters of hematopoietic cell development or expansion.

Engagement of CD24 in vivo had only a very modest effect on the number of CD $45^{+}$Ter119- myeloid lineage cells in the spleen (Figure 1B). There was a minimal impact on the frequency and distribution of cells of the granulocytic and monocytic lineages, with the exception of the Ly6C $\mathrm{C}^{\text {hi }}$ monocyte subset, which showed a very slight incremental increase in frequency (Supplemental Figure 1E).

The above findings suggested that engagement of CD24 in vivo induces a response within the erythroid series, which mimics stress-induced erythropoiesis. Since, in the mouse, extramedullary/stress erythropoiesis is primarily localized to the spleen (10), we examined the impact of splenectomy on the response to $\alpha \mathrm{CD} 24 \mathrm{mAb}$ administration. As Figure $1 \mathrm{H}$ demonstrates, there was a significant $(50 \%)$ reduction in circulating reticulocytes at day 5 after $\mathrm{Ab}$ administration (the peak of the reticulocyte response, Figure 1D) in splenectomized mice. However, this was accompanied by a significant increase in the number of Ter119+ erythroblasts in the BM of splenectomized mice following in vivo CD24 engagement (Figure 1I).

In view of the above findings, it was of interest to formally establish that the in vivo cellular target or targets responding to CD24 engagement were of hematopoietic origin and to ensure that the response observed was directed to $\mathrm{CD} 24$, i.e., not due to an unexpected off-target effect of the administered mAb. To this end, we constructed reciprocal BM chimeras between WT and Cd24 $4^{-/-}$donors in which lethally irradiated WT and $C d 24^{-/-}$recipients were reconstituted with either $C d 24^{-/-}$or WT BM. Following reconstitution, we examined the impact of CD24 engagement in vivo on erythroblast numbers in these chimeric mice as well as in WT and $C d 24^{-/-}$mice. As Figure 2 demonstrates, following CD24 engagement in vivo, splenomegaly and expansion of splenic Ter119+ erythroblasts were observed in treated WT but not $\mathrm{Cd} 24^{-/}$mice (Figure 2A) and in recipients of WT BM but not $C d 24^{-/-} \mathrm{BM}$ (Figure 2, B and C).

$C D 24^{+} C D 8 \alpha^{+}$(cDC1) DCs regulate the initiation of stress sensor-mediated erythropoiesis. The results of the BM chimera analysis established that the stress-type erythropoiesis triggered by CD24 engagement in vivo was dependent on one or more CD24+ cell types of BM origin. To further define the critical target cell type or types, we analyzed CD24 engagement-induced erythropoiesis in the spleens and circulation of mice deficient in or depleted of $\mathrm{CD} 45^{+}$adaptive and innate immune cell types. Adaptive immune cell-deficient recombination-activating gene $1\left(\mathrm{Ragl}^{-/-}\right)$mice displayed the characteristic splenomegaly, with Ter $119^{+}$erythroblast expansion and increase in circulating reticulocytes following $\alpha \mathrm{CD} 24 \mathrm{Ab}$ administration (Figure $3 \mathrm{~A}$ ). Similarly, in vivo depletion of Ly6G $\mathrm{G}^{+}$granulocytes (Figure 3B) or NK cells (Figure 3C) also had no impact on CD24 engagement-induced erythropoiesis.

In contrast, when we acutely depleted CD11 ${ }^{\text {hi }}$ cells by parenteral administration of a low dose of diphtheria toxin (DTx) to Cd11c-DTR mice (where DTR indicates DTx receptor) (ref. 20 and Figure 3D), we markedly diminished CD24 engagement-induced erythropoiesis, as measured by splenic erythroblast numbers (Figure 3E) and circulating reticulocytes (Figure 3F) in a DTx dose-dependent manner (Supplemental Figure 2A). This finding 

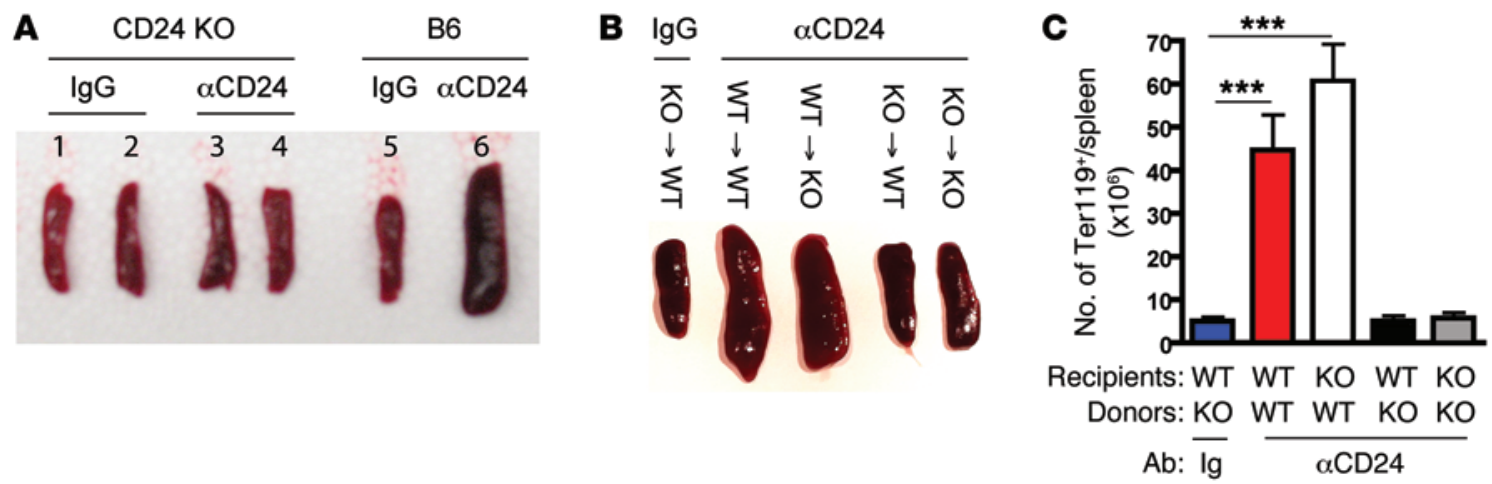

Figure 2. CD24 expressed by cells of hematopoietic origin and not cells in stromal compartments is required for extramedullary stress erythropoiesis following in vivo CD24 engagement. (A) WT or Cd24/-- mice were treated i.p. with M1/69 or lg. At day 5 after Ab treatment, spleens were harvested for examination of gross appearance. (B and $\mathbf{C}$ ) To assess the contribution of CD24-expressing cell types from either hematopoietic or nonhematopoietic compartments, reciprocal BM chimeric mice were established by transferring $2 \times 10^{6}$ donor BM cells derived from either WT or $C d 24^{-/-}$mice into lethally irradiated WT or Cd24 ${ }^{-/-}$recipient mice. Six weeks after BM transplantation, these chimeric mice were infused i.p. with lg or M1/69. At day 5 after treatment, mice were necropsied and evaluated for gross appearance of spleens (B) as well as for erythroid progenitor cells (C) ( $n=4-5 /$ group). Data represent mean \pm SEM. ${ }^{* * *} P<0.001$ (2-tailed, unpaired Student's $t$ test).

suggested that a CD11 $\mathrm{c}^{+}$cell type, possibly a type of DC, might be playing a central role in the stress erythropoiesis stimulated by CD24 engagement. It was therefore of interest to evaluate the expression of CD24 on the surface of DCs (and potentially macrophages) present in the spleen that express a high level of CD11c, as these cells were preferentially depleted by low-dose DTx administration (Figure 3D). As Figure 3G demonstrates, 2 major DC subsets were detected among the CD11 $\mathrm{c}^{\text {hi }}$ splenic DCs, i.e., CD $8 \alpha^{+}$ DCs and $\mathrm{CD}_{11 b^{+}}(\mathrm{cDC} 2)$ DCs $(21,22)$. Of interest, on the whole, the $C D 8 \alpha^{+}$DC subset expressed substantially higher levels of CD24 than did CD11b ${ }^{+}$(cDC2) (or plasmacytoid) DCs (Figure 3G).

Recently, it has been reported that mice genetically deficient in the regulator of myeloid cell lineage commitment transcription factor Batf3 (Batf3 $\left.3^{--}\right)$have a profound selective defect in the generation of splenic $\mathrm{CD} 8 \alpha^{+} \mathrm{DCs}$ and related DC subset(s) in nonlymphoid tissues, e.g., CD103 ${ }^{+}$DCs (ref. 23 and Figure 4A). To assess the contribution of CD $8 \alpha^{+}$DCs to stress erythropoiesis induced by CD24 engagement in the spleen, we analyzed the effect of $\alpha \mathrm{CD} 24 \mathrm{Ab}$ administration in Batf3 $3^{-1-}$ mice. As Figure 4, B and C, demonstrates, stress erythropoiesis triggered by stress sensor engagement was markedly diminished in $\mathrm{Batf}^{-/-}$mice, as measured by reticulocytosis in circulation (Figure $4 \mathrm{~B}$ ) and erythroid progenitor expansion in spleens (Figure 4C). These results suggest that, among $\mathrm{CD} 11 \mathrm{c}^{\text {hi }} \mathrm{DC}$ subsets, $\mathrm{CD} 8 \alpha^{+} \mathrm{DCs}$ likely play a dominant role in regulating stress sensor engagement-dependent extramedullary erythropoiesis in vivo.

To further establish the contribution of DCs in regulating the induction of the initiation of the response of early erythroid progenitors to stress sensor engagement, we adaptively transferred WT CD $24^{+}$BM-derived DCs (BMDCs) into $\mathrm{Cd} 24^{-/-}$mice prior to $\alpha \mathrm{CD} 24 \mathrm{mAb}$ administration. As Figure 4D demonstrates, adoptive transfer of these BMDCs into $C d 24^{-/-}$mice restored expansion of splenic Ter $119^{+}$early erythroid progenitors as well as the corresponding expansion of splenic $\mathrm{CD}_{117}{ }^{+} \mathrm{Kit}^{+}$progenitors (Supplemental Figure 2B) following stress sensor engagement in vivo.

Macrophages have long been appreciated as playing an important role in regulating erythropoiesis under homeostasis and stress $(1,2,7,10)$. Recently, a subset of macrophages displaying CD169 (sialoadhesin [Siglec-1]) found in the marginal zone of the spleen has been reported to be a critical regulator of erythropoiesis, particularly under stress conditions (14). Interestingly, DCs, in particular, those in the CD $8 \alpha^{+}$DC subset, are also found in marginal zones adjacent to red pulp $(24,25)$. It was therefore of interest to analyze splenic CD8 $\alpha^{+}$DCs for the expression of CD169. As Supplemental Figure 2C demonstrates, the splenic CD11 chi DC subsets do not express detectable cell-surface CD169. This finding is consistent with a previous report employing Cd169-DTR mice showing that splenic CD11 $\mathrm{c}^{\text {hi }}$ DC numbers were unaffected by DTx-induced CD169+ macrophage ablation in the spleen (26).

The contribution of macrophages to basal or stress erythropoiesis has been primarily approached by assessing the impact of macrophage depletion, e.g., by chlodronate liposome administration $(3,14)$. Since liposome administration is likely to eliminate both DCs and macrophages, this strategy alone does not show whether the 2 cell types occupy the same or different functional niches during stress erythropoiesis. To ascertain whether CD11chi DCs and resident splenic macrophages function at the same or different phases of the stress erythropoietic response, we evaluated the impact of elimination of CD11 $\mathrm{c}^{\mathrm{hi}}$ DCs (by DTx treatment of Cd11c-DTR mice; ref. 20) at various times after $\alpha \mathrm{CD} 24 \mathrm{Ab}$-induced stress erythropoiesis. As Figure $4 \mathrm{E}$ (and Supplemental Figure 2D) demonstrates, DTx administration at the time of stress erythropoiesis induction, i.e., days -1 to 0 , markedly inhibited the stress erythropoiesis response. However, delay in the elimination of CD11chi DCs, i.e., delay in DTx treatment, by as little as 24 hours after induction of stress erythropoiesis resulted in a normal erythropoiesis response. This finding suggests that $C D 8 \alpha^{+}$DCs act very early in the development of the stress erythropoietic response. In contrast, the elimination of phagocytic cells in vivo (including CD169+ macrophages) by clodronate liposome administration $(3,14)$ when delayed for up to 2 to 3 days still results in a significant inhibition of stress erythropoiesis (Figure 4F). This finding is consistent with the concept that splenic macrophages (including, presumably, CD169+ macrophages) likely have a different role in the evolution and maintenance of stress erythropoiesis than CD11chi CD $8 \alpha^{+}$DCs. 
A

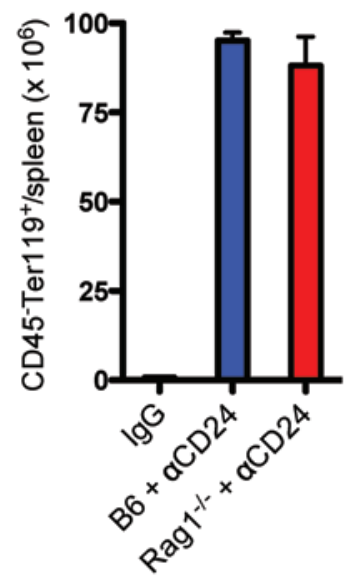

D

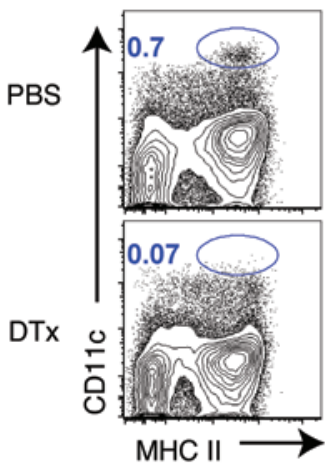

B
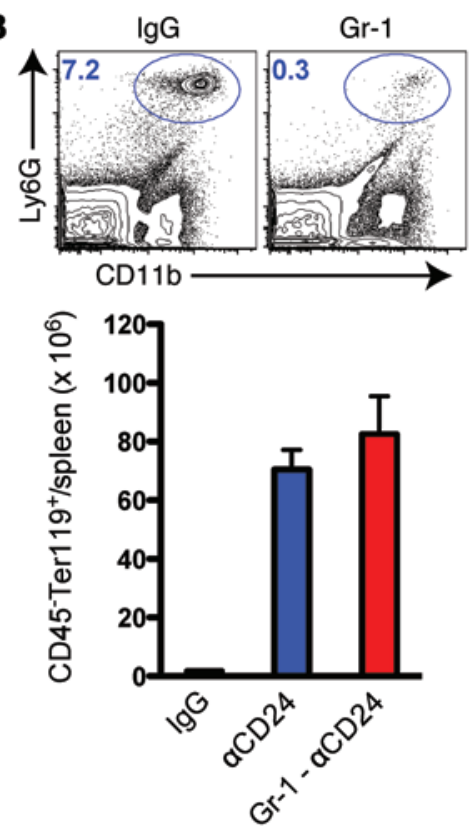

E

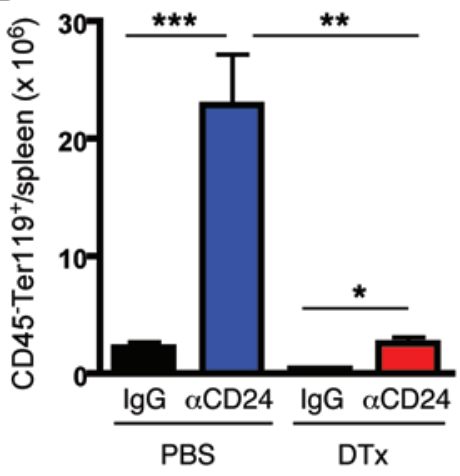

C
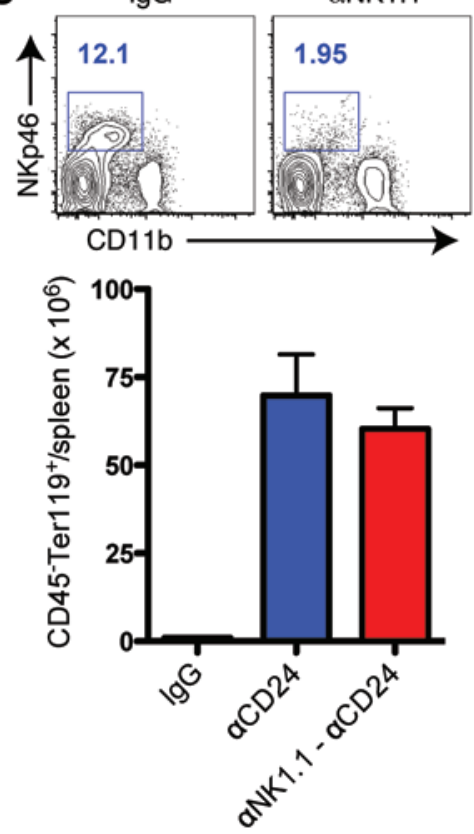

$\mathbf{F}$

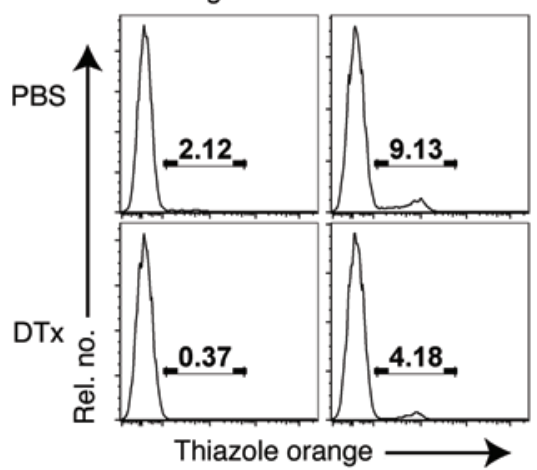

G
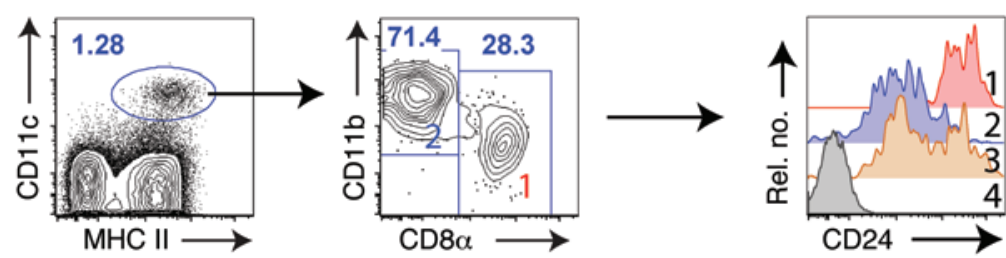

1. $C D 8 \alpha+D C$

2. $C D 11 b+D C$

3. $\mathrm{pDC}$

4. Isotype

Figure 3. Conventional DCs are essential for CD24-mediated extramedullary stress erythropoiesis in vivo. (A-C) Stress erythropoiesis induced by $\alpha$ CD24 mAb treatment occurs independently of lymphocytes, granulocytes, and NK cells. Rag1 ${ }^{-1-}(\mathbf{A})$ or WT mice (B and C) were injected i.p. with $100 \mu \mathrm{g}$ control Ig or M1/69. Ab-administrated mice were depleted of Gr-1+ granulocytes with $100 \mu \mathrm{g}$ of anti-Gr-1 mAbs (B) or anti-Ly6C mAbs (1AG, data not shown) at day 0 . To deplete NK cells, mice received $200 \mu \mathrm{g}$ of anti-NK1.1 (PK136) mAbs at day 0 (C). The depletion efficiency of target cell type in the spleens was confirmed at day 1 by flow cytometry (top panels in $\mathbf{B}$ and $\mathbf{C}$ ). One representative of the frequency of CD45-Ter119+ erythroid progenitors in spleens is shown, as measured by flow cytometry at day 5 after M1/69 treatment ( $\mathbf{A}$ and bottom panels in $\mathbf{B}$ and $\mathbf{C}$ ). Data represent mean $\pm \mathrm{SEM}(n=3-5)$. Cd11c-DTR mice were employed to conditionally deplete CDCs. (D) Upon i.p. DTx administration, DTR-expressing CD11c cells (CDC) were selectively ablated within 24 hours. (E and F) These cDC-ablated mice were infused with Ig or M1/69 4 hours after first DTx administration. Mice were necropsied at day 5 for the gross appearance of the spleens (data not shown), measurement of $\mathrm{CD} 45^{-}$Ter119+ erythroid progenitors in the spleens (E), and percentage of circulating reticulocytes (F) $(n=3-5)$. Data represent mean \pm SEM. ${ }^{*} P<0.05 ;{ }^{* *} P<0.01$; ${ }^{* *} P<0.001$ (2-tailed, unpaired Student's $t$ test). (C) Expression of CD24 among the DC subsets in the murine spleens (left panel); 2 conventional DC (CDC) subsets including CD8 $\alpha^{+} C D 11 b^{-}\left(C D 8 \alpha^{+}\right.$CDC, gate 1) and CD8 $\alpha^{-} C D 11 b^{+}\left(C D 11 b^{+}\right.$CDC, gate 2) (middle panel), and plasmacytoid DCs (CD11 ${ }^{\text {int }}$ Siglec $\mathrm{H}^{+} \mathrm{B} 22 \mathrm{O}^{+}, \mathrm{pDC}$ ) (data not shown). Cell surface CD24 expression was detected by flow cytometry (right panel). Representative data of at least 3 experiments are shown.

Role of $C D 24$ and $C D 8 \alpha^{+} D C$ in the regulation of stress erythropoiesis. Our observations so far suggest that CD11 $\mathrm{c}^{\text {hi }}$ DCs (likely primarily $\mathrm{CD} 8 \alpha^{+} \mathrm{DCs}$ ) play a central role in the induction of erythropoiesis following in vivo engagement of the stress sensor CD24 (presumably displayed by this DC subset) and suggest that this cell type may serve a corresponding role in the induction of erythropoiesis following other forms of stress. It is noteworthy that both $C d 24^{-/}$mice and CD $8 \alpha^{+}$ DC-deficient Batf $3 \%$ mice have Hb and HCT in the normal range, 
A

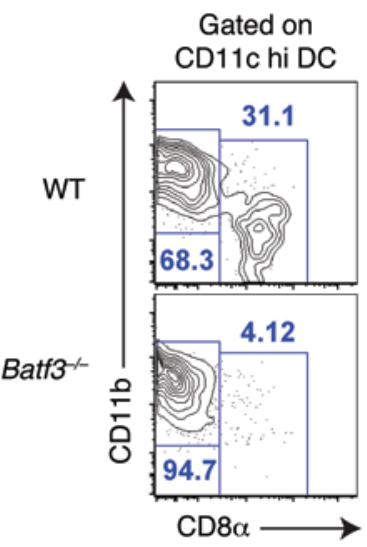

D

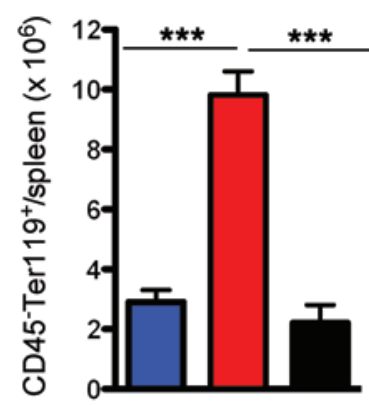

Recipient mice

(CD24): -/- -/- -/-

Donor cell - DC

(CD24): $-/-\quad+/+\quad+/+$

$\alpha \mathrm{CD} 24 \mathrm{Ab}:++$
B

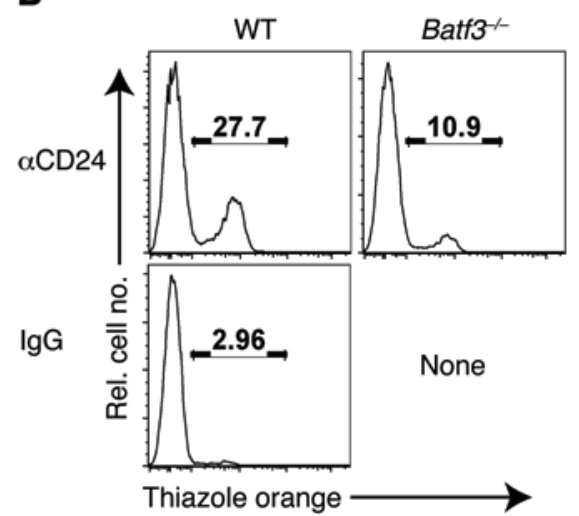

E DTx

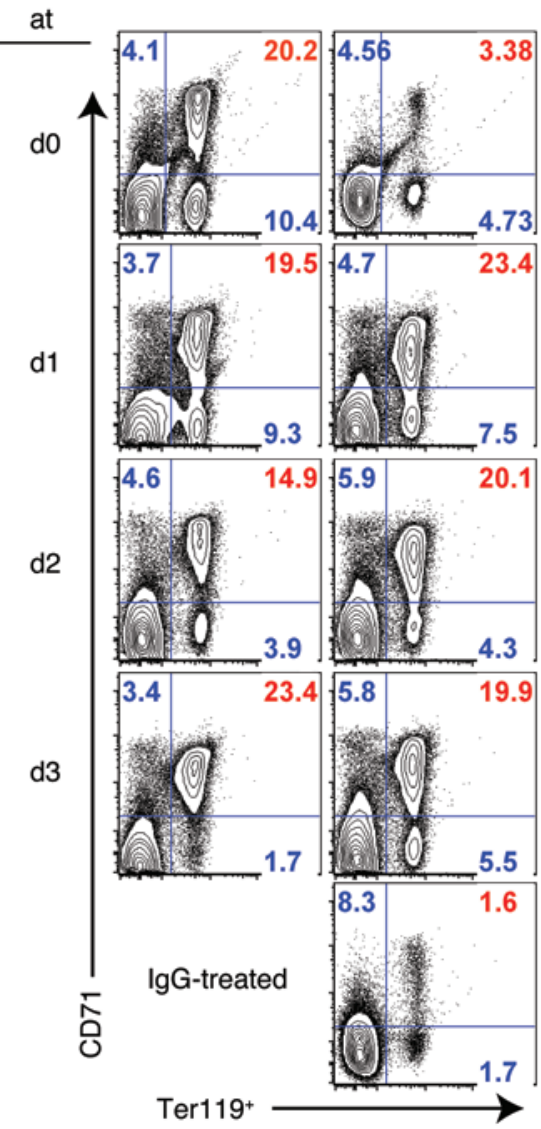

C

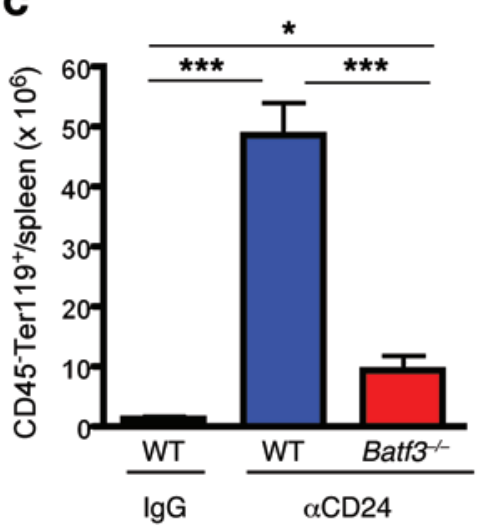

F Liposome admin. Control Clodronate

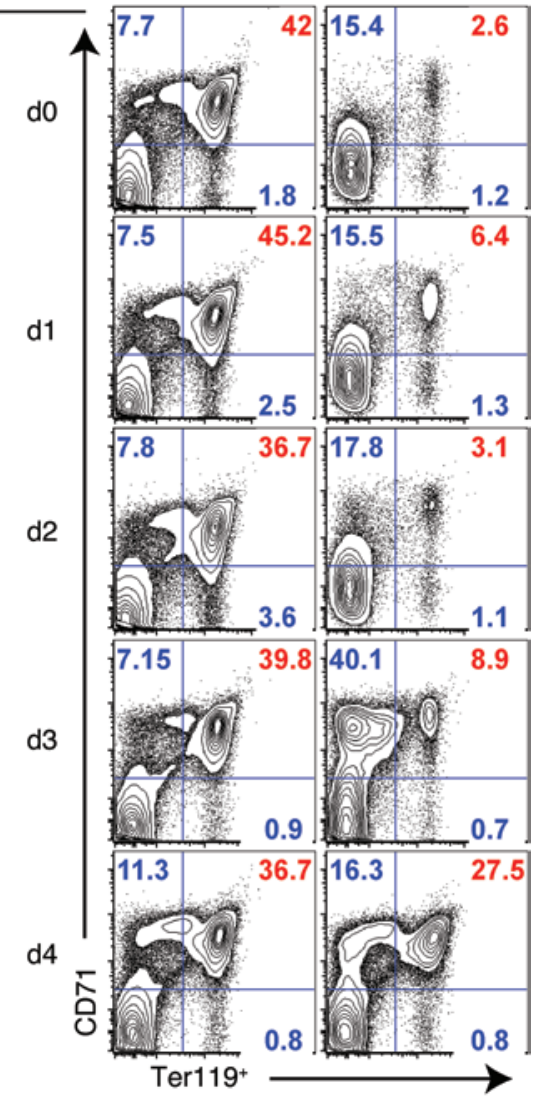

Figure 4. $\mathrm{CD24}+\mathrm{CD8} \boldsymbol{\alpha}^{+} \mathrm{DCs}$ regulate the initiation of CD24-mediated stress erythropoiesis. (A) Mice lacking transcriptional factor Batf3 gene (Batf3 $3^{-1-}$ mice) were examined for the selective deficiency of $\mathrm{CD} 8 \alpha^{+} \mathrm{CDC}$ subsets in the spleen. (B and C) WT and Batf3-- mice were injected i.p. with $100 \mu \mathrm{g}$ control Ig or M1/69. After 5 days, the percentage of reticulocytes in the blood (B) and absolute number of CD45-Ter119+ erythroid progenitors in the spleens (C) were assessed by flow cytometry $(n=4-6)$. Data represent mean \pm SEM. ${ }^{*} P<0.05$; ${ }^{* * *} P<0.001$ (Student's $t$ test). (D) BMDCs prepared from either WT or $C d 24^{-/}$mice were transferred i.v. into $C d 24^{--}$mice prior to $\alpha \mathrm{CD} 24 \mathrm{mAb}(\mathrm{M} 1 / 69)$ treatment. At day 5 after treatment, late erythroid progenitors (CD45Ter119+) in the spleens were enumerated ( $n=4-5 /$ group). ${ }^{* *} P<0.001$. (E) $C D C$ in DTR Tg mice were conditionally and selectively eliminated at the indicated days after $\mathrm{M} 1 / 69$ treatment. These $\mathrm{CDC}$-ablated, Ab-treated mice were necropsied at day 5 for the measurement of $\mathrm{CD} 45^{-}$-Ter119+ erythroid progenitors in the spleens. Data shown are representative of at least 3 independent experiments. (F) Phagocytic cells were eliminated in vivo by clodronate liposome administration at the indicated days after M1/69 treatment. These phagocyte-depleted, Ab-treated mice were necropsied at day 5 for the measurement of CD45-Ter119+ erythroid progenitors in the spleen by flow cytometric analyses. Data shown are representative of at least 3 independent experiments.

i.e., are not anemic (Supplemental Figure 3A). Therefore, any contribution of CD24 and particularly CD8 $\alpha^{+}$DCs would be demonstrable primarily following stress. To explore this possibility, we subjected $\mathrm{Cd} 24^{--}$and Batf $3^{--}$mice (and age-matched controls) to stress in the forms of (a) anemia induced by cisplatin treatment, (b) chronic hypoxia following housing of mice under conditions of normobaric hypoxia $\left(10 \% \mathrm{O}_{2}\right)$ over 2 weeks, and (c) anemia induced by phlebotomy. Under these stress conditions, we analyzed the time course of the response to 

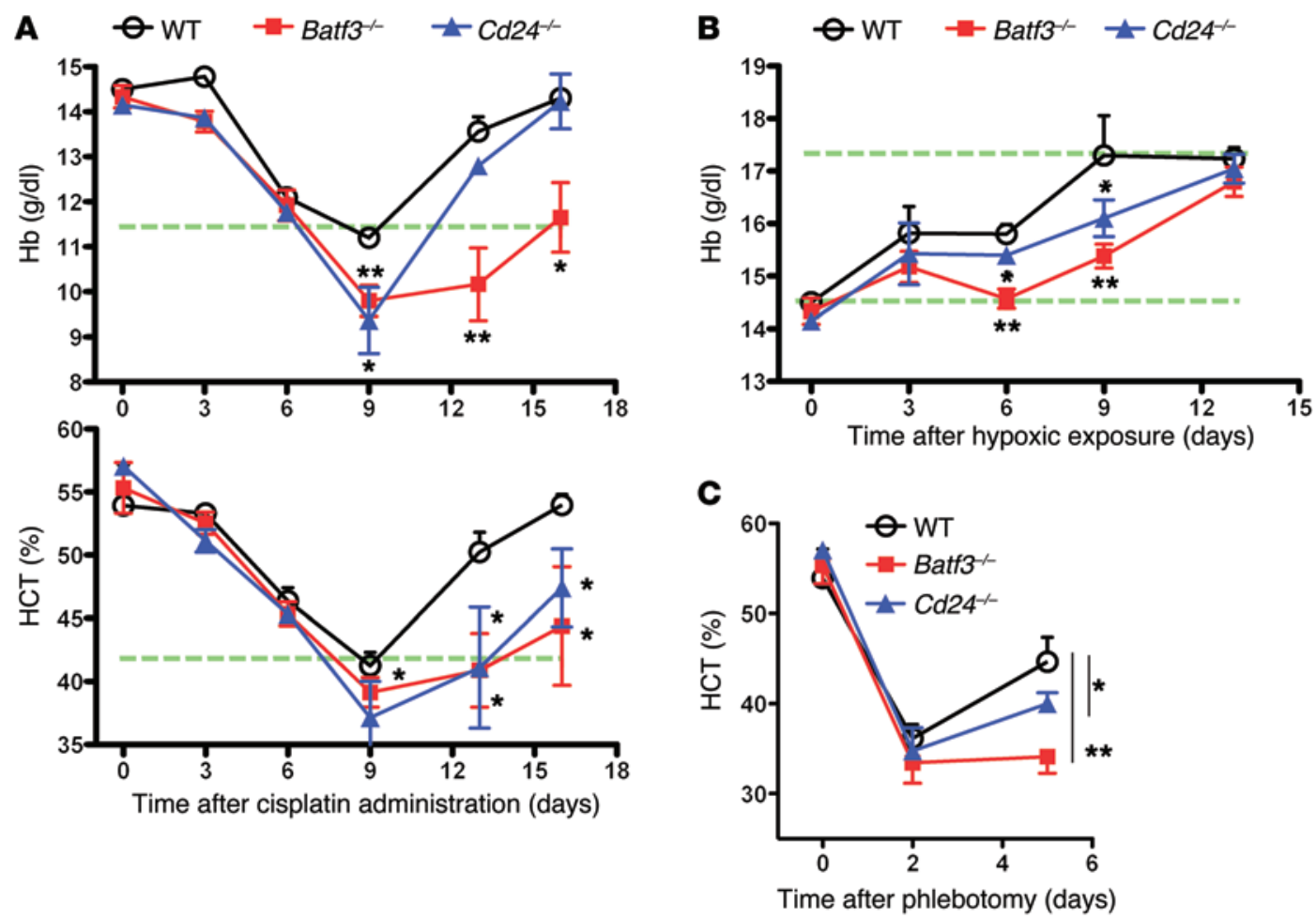

Figure 5. The impact of CD24 and CD8 $\alpha^{+}$DC subsets on the regulation of erythropoiesis under stressed conditions in vivo. (A) WT, Batf $3^{-/ /}$, and $C d 24^{-/-}$mice

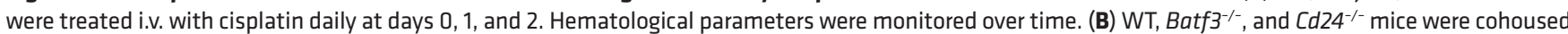
in hypoxic chambers (10\% oxygen). Serum Hb levels were measured over time. (C) WT, Batf3 ${ }^{-1-}$, and $C d 24^{-1-}$ mice were bled daily at days 0,1 , and 2 . HCT levels in the phlebotomized mice were monitored over time. Data represent mean \pm SEM ( $n=4-6$ /genotype) of 2 independent experiments. ${ }^{*} P<0.05$; ${ }^{* *} P<0.01$.

these stresses at the levels of $\mathrm{Hb}$ and HCT in WT, $\mathrm{Cd} 24^{-/}$, and Batf3 $3^{-/}$ mice. As Figure 5A demonstrates, Batf $3^{-/-}$mice showed significantly prolonged and delayed responses to the anemia induced by cisplatinbased therapy, as measured by $\mathrm{Hb}$ and HCT, for up to 17 days. Comparable results were obtained for $\mathrm{Batf}^{-/-}$mice when stress erythropoiesis was induced by hypoxia (Figure $5 \mathrm{~B}$ for $\mathrm{Hb}$ ) or by phlebotomy resulting in acute anemia (Figure $5 \mathrm{C}$ for $\mathrm{HCT}$ ). Similarly, $\mathrm{Cd} 24^{-/-}$mice, when compared with WT mice, also exhibited a comparable delayed response to these forms of stress as well as to phenylhydrazineinduced (PHZ-induced) hemolytic anemia (Supplemental Figure 3B), but absence of the CD24 stress sensor had a less pronounced effect than absence of the sensor-bearing DC subset. These observations further suggest an important role of DCs and, in particular, the CD $8 \alpha^{+}$ DC subset (and to a lesser extent the CD24 sensor molecule) in the regulation of erythropoiesis following a natural stress stimulus.

$C D 8 \alpha^{+}$DCs, Kit, and erythroblast expansion. CD117 (the cell surface protein tyrosine kinase Kit) is well established as a marker for hematopoietic stem cells and progenitors including erythroid progenitors $(27,28)$. Effective erythropoiesis is also reported to depend on engagement of Kit by its ligand, SCF (27). We determined that stress erythropoiesis induced by CD24 engagement was Kit dependent, as treatment of mice with the Kit-signaling inhibitor imatinib (also known as Gleevec) at the time of $\alpha \mathrm{CD} 24 \mathrm{Ab}$ administration markedly inhibited erythroblast expansion and the increase in circulating reticulocytes (Figure 6, A and B). In keeping with this finding, CD24 engagement-dependent erythropoiesis was likewise significantly diminished in WT mice that had received Kit-blocking mAbs (ACK2) and Kit-mutant W/Wv mice (Figure 6C).
To further explore the role of Kit/SCF interaction following CD24 engagement-dependent stress erythropoiesis, we examined the impact of in vivo CD24 engagement on $\mathrm{Kit}^{+}$cell proliferation. We could detect a small number of $\mathrm{Kit}^{+} \mathrm{Ter} 119^{-} \mathrm{CD} 71^{-}$cells within the CD45- population in the spleens of adult mice that we considered likely to be early erythroid progenitors (ref.10 and Supplemental Figure 4). Upon CD24 ligation, these cells rapidly, i.e., within 24 hours, underwent robust proliferative expansion far exceeding background homeostatic proliferation (Figure 6D) as measured by BrdU uptake and Ki-67 nuclear staining, respectively (Figure $6 \mathrm{E}$ ). This proliferative expansion in response to $\mathrm{CD} 24$ engagement was effectively abrogated by $\alpha$ Kit $\mathrm{mAb}$-mediated in vivo blockade (Figure 6, F and G). More importantly, consistent with the critical role of $\mathrm{CD} 8 \alpha^{+}$DCs in the induction of stress erythropoiesis, CD24 engagement in $\mathrm{CD} 8 \alpha^{+} \mathrm{DC}$-deficient $\mathrm{Batf3}^{-/-}$mice poorly triggered $\mathrm{Kit}^{+} \mathrm{CD} 45^{-}$Ter119- ${ }^{-} \mathrm{CD} 71^{-}$cell proliferation at this early time point, i.e., within 24 hours of in vivo CD24 engagement (Figure 6H).

Although in vivo Kit signaling inhibition (i.e., imatinib) or Kit blockade (i.e., ACK2 mAb) suppressed the proliferative expansion of early erythroid progenitors and the subsequent increase in circulating reticulocytes in response to stress erythropoiesis induction by CD24 engagement, Kit blockade did not suppress stressinduced EPO production. Indeed, circulating levels of EPO were elevated in $\alpha \mathrm{CD} 24 \mathrm{Ab}$-treated animals undergoing Kit blockade compared with Ab-treated control WT animals (Supplemental Figure 5A). Thus, expansion of early erythroid progenitors following stress was dependent on Kit signaling and this expansion was not enhanced in the face of elevated circulating EPO. 
A
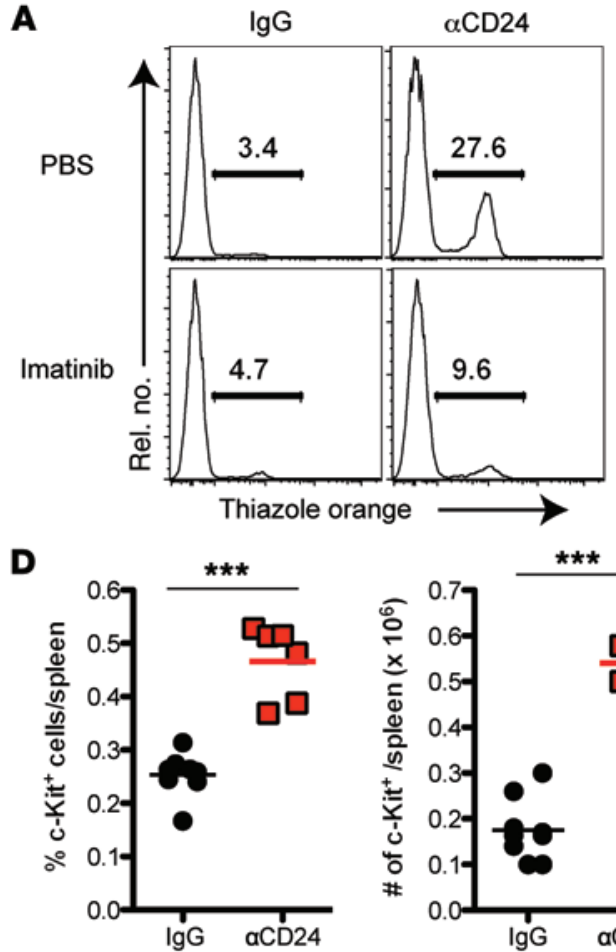

$\mathbf{F}$
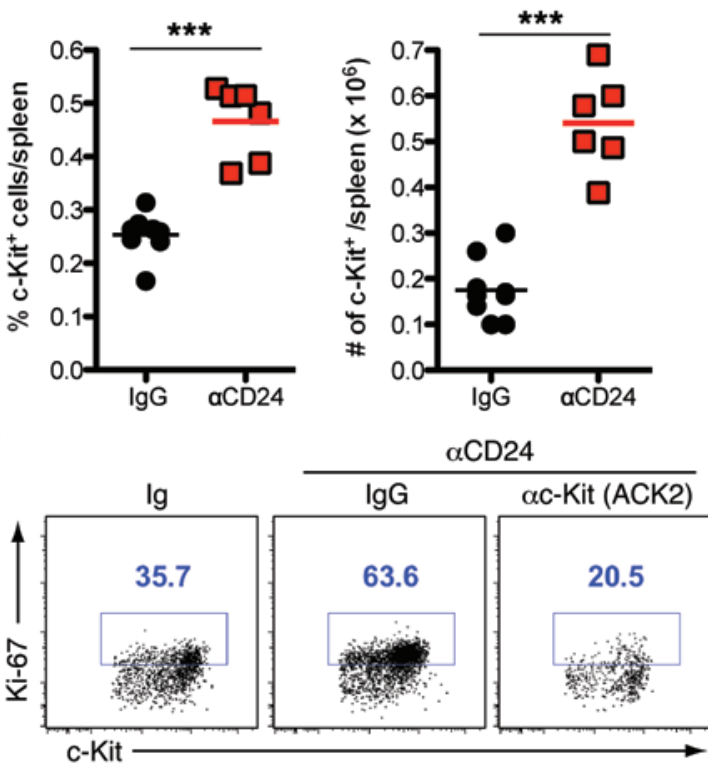

G

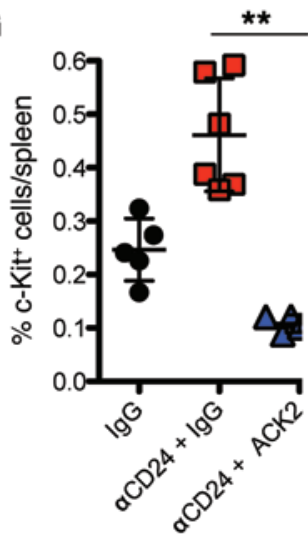

B

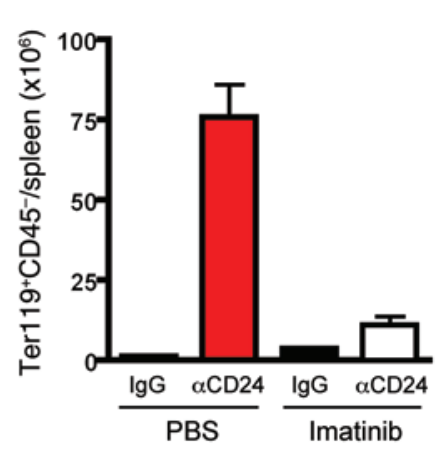

C

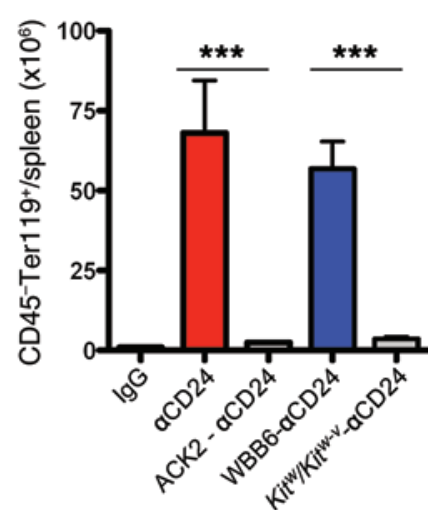

E

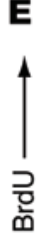

$\lg$
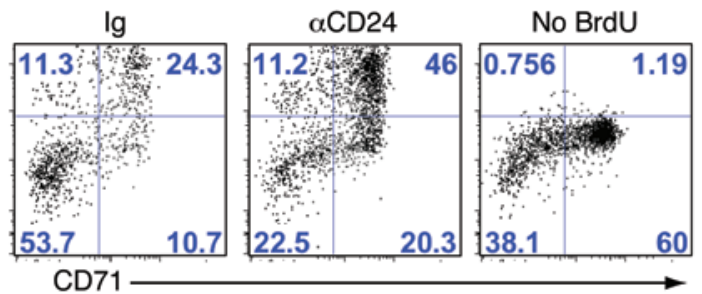

CD71

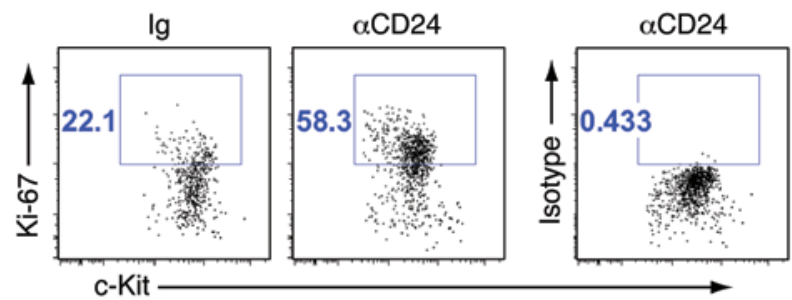

H

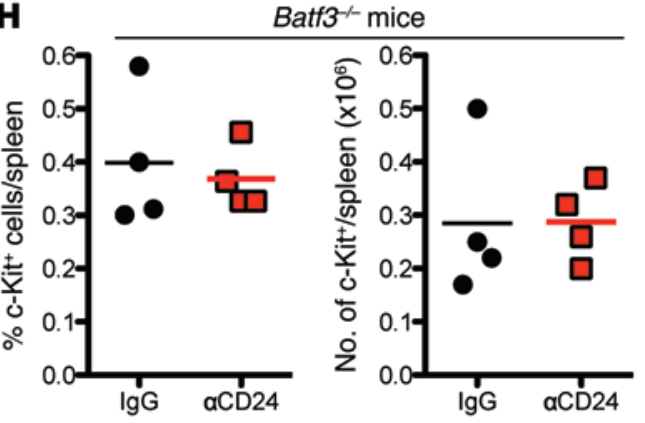

Figure 6. The SCF/Kit signaling axis is critical for CD24-induced stress erythropoiesis at extramedullary site. (A and B) The pharmacological inhibitor of Kit signaling, imatinib, was administrated ( $1 \mathrm{mg} / \mathrm{kg}$ ) i.p. daily for 4 days into M1/69-treated WT mice. At day 5, reticulocytes in the blood (A) and the expansion/accumulation of CD45-Ter119+ erythroid progenitors in the spleen (B) were examined. Data represent mean \pm SEM $(n=4)$ of 2 independent experiments. (C) WT B6 mice were treated i.p. with Kit signaling blocking Abs (clone ACK2) at days 0 and $1\left(200 \mu \mathrm{g} /\right.$ each injection) after M1/69 treatment. In addition, Kit mutant (Kit $\left.{ }^{w} / K^{2} t^{w-\nu}\right)$ or controls (WBB6 background) were treated with M1/69 Abs. At day 5 after M1/69 treatment, mice were examined for percentage of reticulocytes in peripheral blood (data not shown), gross appearance of spleens (data not shown), and numbers of CD45-Ter119+ erythroid progenitors in the spleen. Data represent mean \pm SEM $(n=4)$ combined from 2 independent experiments. ${ }^{* * *} P<0.001$. ( $D$ and $\left.\mathbf{E}\right)$ M1/69 treatment promotes the expansion of Kit ${ }^{+} C D 45^{10 /-C D 71+/-}$ cells in the spleen. WT mice were injected i.p. with $100 \mu \mathrm{g}$ control Ig or M1/69. Total Kit ${ }^{+}$CD45 $5^{10 /-}$ cells in the spleen were examined 1 day after Ab treatment (D). To determine whether $\mathrm{Kit}^{+} \mathrm{CD} 45^{10 /-} \mathrm{CD} 71^{+/-}$cells undergo proliferative expansion, M1/69-treated mice were fed i.p. with nucleic acid analog, BrdU, to label proliferating cells 1 hour prior to harvest. Kit ${ }^{+}$erythroid progenitors were examined at 24 hours after $\mathrm{Ab}$ treatment for active uptake of BrdU (upper panels) in combination with staining for a proliferation-associated nuclear antigen, Ki-67 (bottom panels) (E). Data represent at least 3 independent experiments. (F and C) M1/69-treated WT B6 mice were i.p. injected with Kit-blocking mAbs (clone ACK2) at day $0(200 \mu \mathrm{g})$. Twenty-four hours later, splenic Kit ${ }^{+}$CD45 $5^{\text {lo/- }}$ cells were examined for Ki-67 nuclear staining (F)

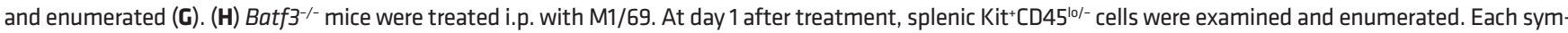
bol shown in $\mathbf{D}, \mathbf{G}$, and $\mathbf{H}$ represents an individual mouse; small horizontal lines indicate the mean. ${ }^{* *} P<0.01$; ${ }^{* * *} P<0.001$ (2-tailed, unpaired Student's $t$ test). 
A

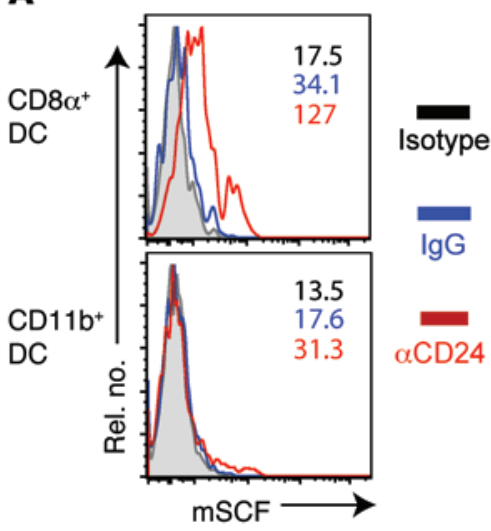

D

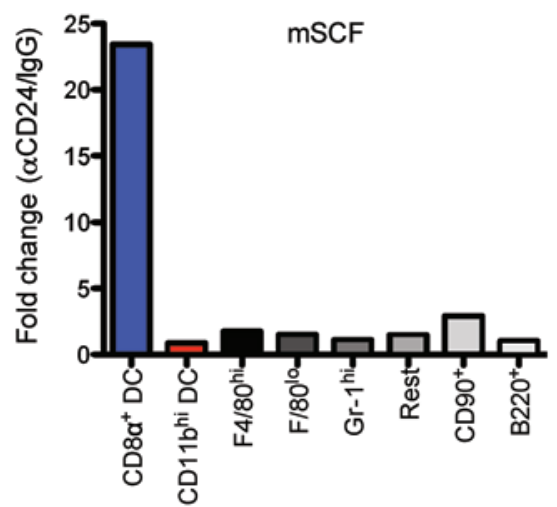

B

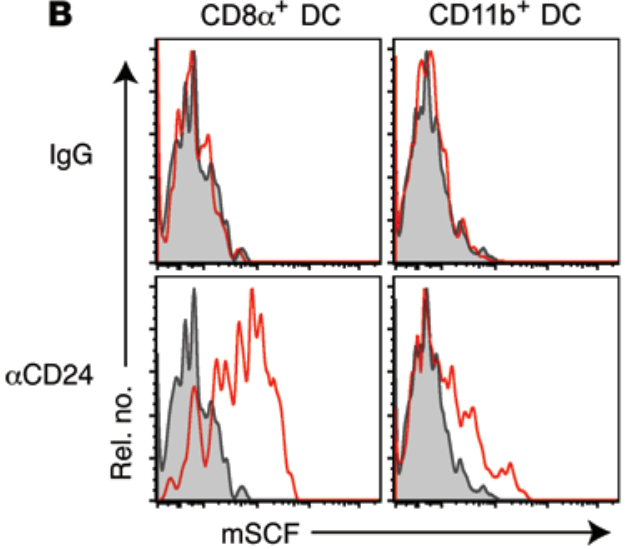

E

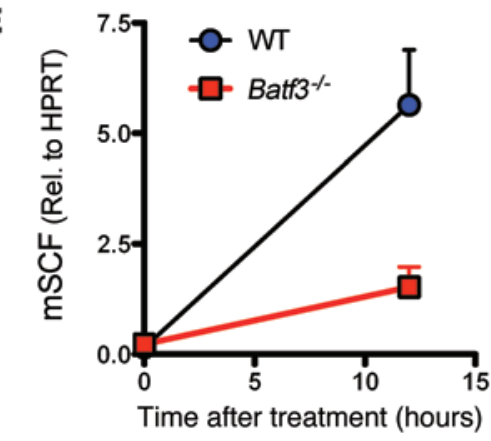

C

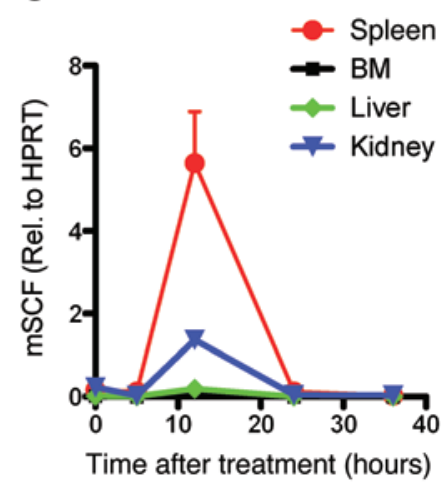

Figure 7. Splenic CD8 $\alpha^{+}$DCs are a major source for SCF produced after CD24 engagement. (A) Single-cell suspensions prepared from spleens of naive mice were incubated with control lg or M1/69 for 12 hours in vitro and examined for mSCF expressed by splenic DC subsets. (B) WT B6 mice treated with M1/69 in vivo were harvested at 12 hours after Ab treatment. Splenic DC subsets were examined for the expression of $\mathrm{mSCF}$. Data shown in $\mathbf{A}$ and $\mathbf{B}$ represent at least 3 independent experiments. (C) At various times after M1/69 treatment, the indicated organs were harvested, and $m S C F$ mRNA expression levels were examined. Data represent mean \pm SEM $(n=3-5)$. (D) WT B6 mice were treated i.p. with M1/69. At 12 hours after Ab treatment, the indicated cell types in the spleen were individually FACS sorted. Expression of Scf mRNAs was examined and presented as fold change compared with those from Ig-treated mice (pooled from 4 mice/sorting). Data represent 2 independent experiments. (E) WT and Batf3 $3^{-/-}$mice were treated i.p. with M1/69. Spleens were harvested at 12 hours after Ab treatment. Scf mRNA expression levels were measured. Data represent mean \pm SEM ( $n=3-5 / g e n o t y p e)$.

The elevated level of circulating EPO following Kit blockade was likely due to the failure to generate sufficient numbers of EPO-sensitive erythroblast progenitors in the spleen capable of consuming this growth factor. Indeed, a similar elevated level of circulating EPO was detected in splenectomized $\alpha \mathrm{CD} 24 \mathrm{Ab}$-treated WT mice (Supplemental Figure 5B). These data suggest that, while EPO production is necessary to drive erythroid progenitor differentiation into reticulocytes/rbc, EPO-independent activation and expansion of early Kit ${ }^{+}$progenitors is also necessary for effective induction of stress erythropoiesis.

$C D 8 \alpha^{+} D C s$ and the expression of Kit ligand. The above results are consistent with a well-established role of Kit signaling in regulating erythropoiesis, although a link between Kit signaling, in particular, the expression of the ligand for Kit, i.e., SCF, and DCs has not been formally established. In light of the above findings, it was of interest to determine whether engagement of CD24 on splenic $\mathrm{CD} 11 \mathrm{c}^{+}$cells and, in particular, on $\mathrm{CD} 8 \alpha^{+}(\mathrm{cDC} 1) \mathrm{DCs}$ affected the expression of SCF by the DCs.

As Figure 7A illustrates, incubation of naive splenic DCs with $\alpha \mathrm{CD} 24 \mathrm{Abs}$ in vitro resulted in upregulation of expression of the membrane-bound variant of SCF $(\mathrm{mSCF})$ on splenic $\mathrm{CD} 11 \mathrm{c}^{+}$ cells with the greatest upregulation observed within the CD $8 \alpha^{+}$ DC subset. We also observed upregulation of MSCF among DCs derived from $\mathrm{BM}$ cell progenitors in cultures upon CD24 ligation, albeit at a reduced level compared with splenic CD $8 \alpha^{+}$DCs (Supplemental Figure 6, A and B). Importantly, when we analyzed mSCF expression on splenic DCs directly ex vivo at 12 hours (Figure $7 \mathrm{~B}$ ) and 24 hours (data not shown) after in vivo $\alpha \mathrm{CD} 24$ $\mathrm{Ab}$ administration, we also detected upregulation of MSCF on the surface of DCs stimulated in vivo. This was again most pronounced in the CD8 $\alpha^{+}$DC subset (Figure 7B). These data collectively suggest that $\mathrm{CD} 8 \alpha^{+}$DCs found in the murine spleen likely serve as a major sensor of stimuli that evoke stress erythropoiesis in part by responding to stress by upregulating expression of a critical molecular cue, SCF, for the Kit ${ }^{+}$erythroid progenitors to enter cell cycle and undergo initial proliferative expansion.

To further investigate the role of $\mathrm{CD} 24$ and the splenic $\mathrm{CD} 8 \alpha^{+}$ DCs in stress erythropoiesis, we next analyzed the SCF gene expression (i.e., transcripts for both $\mathrm{mSCF}$ and secreted form $[\mathrm{sSCF}])$ in various tissues/organs over time. As demonstrated in Figure $7 \mathrm{C}, \mathrm{mSCF}$ is highly expressed in the spleen and to a lesser extent in the kidney, with levels in both organs peaking at around 
A

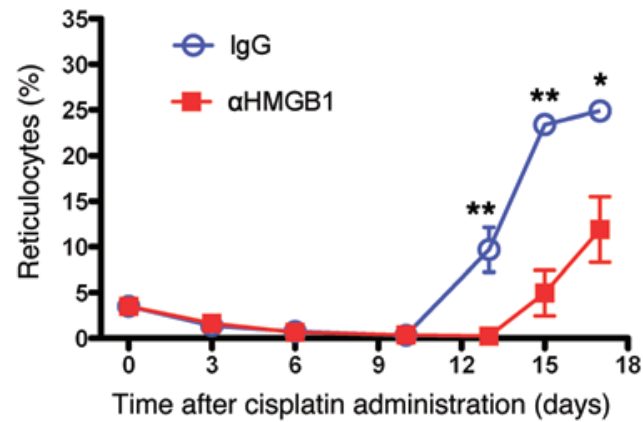

B

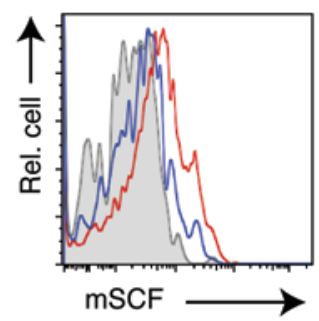

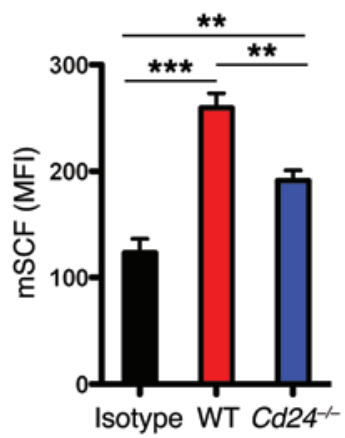

Figure 8. HMGB1/CD24 sensor system plays an important role in stress erythropoiesis. (A) Mice were treated i.v. with cisplatin daily at days 0,1 , and 2. The cisplatin-treated mice were administrated i.p. with $\alpha$ HMGB1 blocking mAbs at days 1, 2 and 3 after cisplatin treatment. Reticulocytes in the peripheral blood were analyzed. Data shown represent mean \pm SEM of pooled mice of at least 2 independent experiments $(n=3-5)$. (B) Splenocytes isolated from WT or $C d 24^{--}$mice were incubated with HMGB1 $(20 \mu \mathrm{g} / \mathrm{ml})$ for 14 hours in cultures. SCF upregulation by splenic DC subsets was monitored by flow cytometry. Data represent mean \pm SEM of 4 to 5 samples per genotype of 2 independent experiments. ${ }^{*} P<0.05 ;{ }^{* *} P<0.01$; ${ }^{* *} P<0.001$ (unpaired Student's $t$ test).

12 hours after CD24 engagement and rapidly declining thereafter. Similarly, we also detected SSCF at the early time points exclusively in the spleen, with a return to basal levels within 36 hours (Supplemental Figure 6C). In addition, upregulation of $B M P 4$ gene expression was detectable in spleens, but was modest, i.e., less than 2 -fold (Supplemental Figure 6D). To identify the potential cellular source or sources for SCF, we fractionated total splenocytes into multiple cellular fractions either before or 12 hours after in vivo $\alpha \mathrm{CD} 24 \mathrm{Ab}$ treatment. Strikingly, CD24 engagement-dependent SCF upregulation was primarily restricted to $\mathrm{CD} 8 \alpha^{+}$cells, with $\mathrm{F} 4 / 80^{\text {hi }}$ macrophages and other splenic cell types displaying minimal upregulation of the Kit ligand (Figure 7D and Supplemental Figure 6E). In keeping with these results, there was minimal upregulation of SCF expression in the spleens of Batf3 $3^{-1-}$ mice in response to in vivo CD24 engagement at this time point (Figure 7E and Supplemental Figure 6F). In sum, these findings suggest that CD $8 \alpha^{+} \mathrm{DCs}$ likely serve as a major source of SCFs (mSCF and potentially sSCF) required to trigger $\mathrm{CD} 24$-dependent stress erythropoiesis.

$C D 11 c^{+}$cells and the regulation of EPO production. Along with Kit, EPO plays a central role in erythropoiesis under both homeostasis and stress $(9,29,30)$. As noted above (Figure $1 \mathrm{~F}$ and Supplemental Figure 5), engagement of CD24 in vivo results in a transient elevation of EPO in the circulation. This finding was initially surprising, as the primary stimulus for increased EPO release is hypoxemia/anemia. Nevertheless, elevated EPO production would be expected to be necessary for the induction of stress-type erythropoiesis. However, we observed that engagement of CD24 in vivo resulted in upregulation of EPO gene expression in the kidneys (Supplemental Figure 7A). In keeping with this result, we found that the expression of the genes encoding the critical regulators of EPO gene expression in the kidneys, HIF- $1 \alpha$ and, in particular, HIF-2 $\alpha$, were likewise upregulated in the kidneys following in vivo CD24 engagement (Supplemental Figure 7B).

Of note, we found that the transient elevation of serum EPO levels in response to in vivo CD24 engagement also required a DC-like cell type, as in vivo depletion of CD11 ${ }^{+}$cells (by DTx treatment of Cd11c-DTR mice) at the time of $\alpha \mathrm{CD} 24 \mathrm{Ab}$ administration markedly diminished the levels of circulating EPO (Supplemental Figure 7C). While these findings suggested a role for CD11 $\mathrm{c}^{+}$cells in the regulation of CD24-stimulated EPO production, the control of EPO production following CD24 engagement was not dependent on a response from splenic $\mathrm{CD} 11 \mathrm{c}^{+} \mathrm{CD} 8 \alpha^{+} \mathrm{DCs}$, since EPO release into the circulation in response to $\mathrm{CD} 24$ engagement was also observed in treated splenectomized mice (Supplemental Figure 5B). On the other hand, neutralization of circulating EPO in $\alpha \mathrm{CD} 24$-treated mice had only a modest effect on the initial proliferative expansion of $\mathrm{Kit}^{+}$early erythroid progenitors in the spleen at day 2 after CD24 engagement (Supplemental Figure 8A), but in vivo EPO neutralization did, as expected, markedly decrease the total accumulation of Ter $119^{+}$erythroid lineage cells in the spleen by day 5 after in vivo CD24 stimulation (Supplemental Figure 8B).

Alarmin recognition and the induction of stress sensor-dependent erythropoiesis. We reasoned that in vivo engagement of the CD24 stress sensor by Abs resulting in the induction of erythropoiesis presumably mimicked the effect of engagement of this receptor by one or more natural ligands produced (or released) in response to stress, e.g., damage-associated pattern molecules (DAMPs) (31). To further explore this possibility, we evaluated the role of one such CD24 ligand, HMGB-1, by administering $\alpha$ HMGB1 mAbs into WT mice undergoing cisplatin treatment (see Figure 5A). Interestingly, the in vivo blockade of HMGB1 significantly delayed the onset of reticulocyte rebound (Figure $8 \mathrm{~A}$ ) and impaired the regain of the levels of $\mathrm{Hb}$ and HCT (Supplemental Figure 9, A and B) and weight recovery (data not shown), suggesting a likely role for HMGB1 as one potential regulator of stress erythropoiesis induction. Of note, we were unable to stimulate reticulocytosis (or serum EPO elevation) by parenteral administration of a single dose of recombinant HMGB1. This was not surprising, as the structural variant of HMGB1 that confers optimal stimulation of stress erythropoiesis in vivo is not defined. Treatment of $\mathrm{CD} 24^{+} \mathrm{CD} 8 \alpha^{+} \mathrm{DCs}$ in vitro with recombinant HMGB1, however, did result in increased SCF expression on these cells, induction of which was significantly reduced in treated CD24-CD8 $\alpha$ DCs (Figure $8 \mathrm{~B}$ ).

Response of human cells to stress sensor engagement. In contrast to what occurs in the mouse, stress-induced erythropoiesis in the human is far less understood, but is believed to be 
A
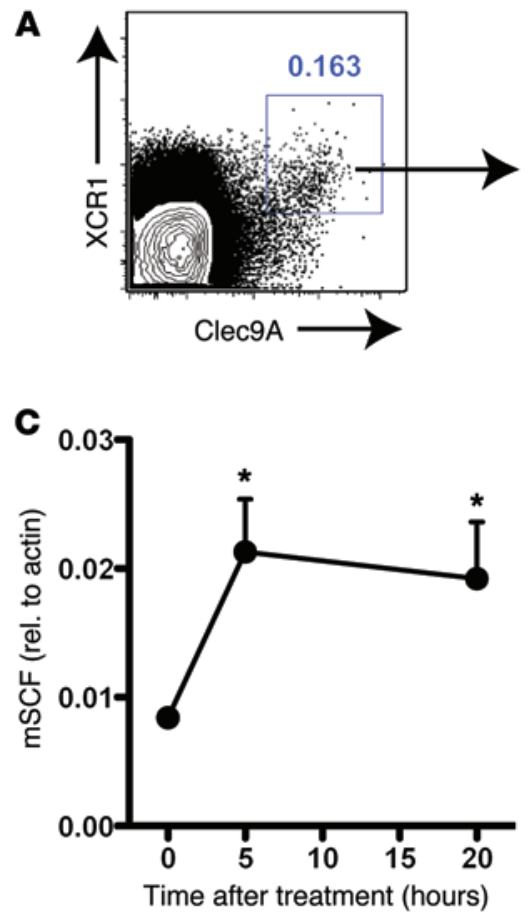
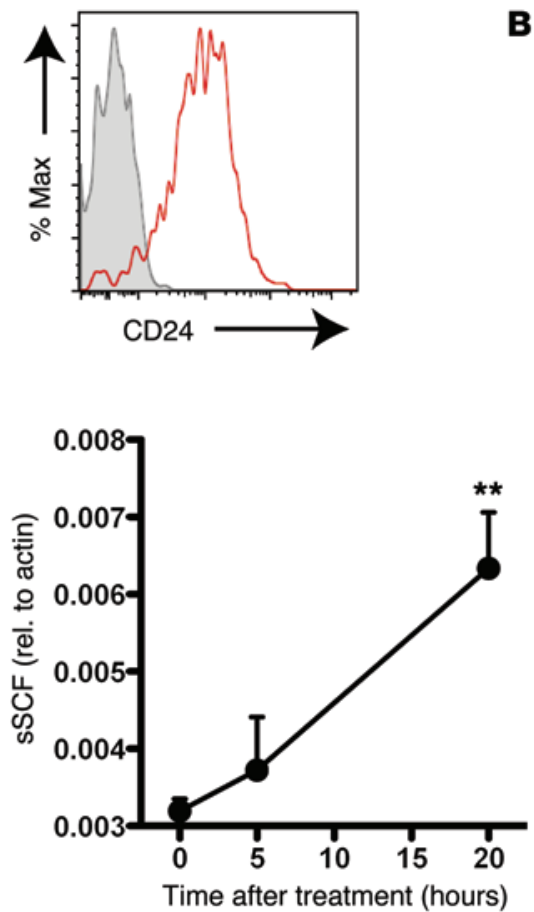

B

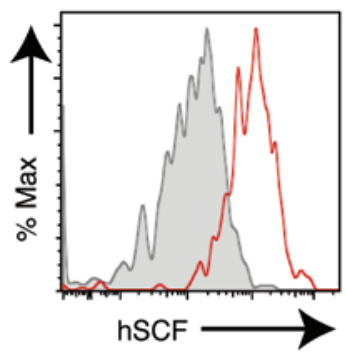

D

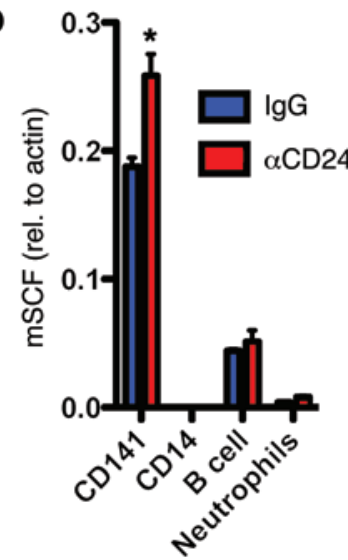

Figure 9. SCF production by human XCR1+CLEC9A ${ }^{+}$DCs in response to CD24 engagement in vitro. (A and $B$ ) Human $B D C A 3^{+}\left(X C R 1^{+} C L E C 9 A^{+}\right) D C s$ were identified from fresh human BM aspirates. Surface expression of CD24 by this fresh DC subset was analyzed (A). BM cells were incubated overnight with 2 different monoclonal Abs to human CD24 ( $\alpha$ hCD24), eBioSN3 and ML5, and the expression of human mSCF by XCR1+CLEC9A DCs was examined (B). Data shown in $\mathbf{A}$ and $\mathbf{B}$ represent at least 3 independent experiments. (C and $\mathbf{D})$ Human BMDCs were generated from human BM aspirates. Expression of SCF mRNA by human BMDCs was examined at day 9 by qRT-PCR (C). Fresh human BM aspirates were FACS sorted into at least 4 subpopulations. The sorted leukocyte populations within the BM were incubated overnight with $\alpha$ hCD24 (clone eBioSN3). Expression of Scf RNA was evaluated after overnight stimulation by qRT-PCR (D). Data in $\mathbf{C}$ and $\mathbf{D}$ represent mean \pm SEM of triplicates of at least 2 independent experiments. ${ }^{*} P<0.05$; ${ }^{* *} P<0.01$ ( 2 -tailed, unpaired Student's $t$ test).

largely (but not exclusively) restricted to the BM (10). In view of the above findings in the mouse, it was of interest to determine whether engagement of CD24 displayed on DCs in the human and, in particular, on the human counterpart of the murine $\mathrm{CD} 8 \alpha^{+}$(cDC1) DCs likewise triggered the upregulation of SCF expression. The human counterpart on the murine splenic $\mathrm{CD} 8 \alpha^{+}$(cDC1) DCs (15) is currently considered to be the human CD $141^{+} \mathrm{XCR}^{+} \mathrm{CLEC} 9 \mathrm{~A}^{+}$DC subset $(16,17)$. To explore the impact of CD24 engagement on SCF expression in the response of human BM cells, we first analyzed CD24 expression in $\mathrm{XCR} 1^{+} \mathrm{CLEC} 9 \mathrm{~A}^{+} \mathrm{DC}$ in fresh human $\mathrm{BM}$ aspirates. We identified among BM aspirate cells a small population of $\mathrm{XCR}^{+} \mathrm{CLEC} 9 \mathrm{~A}^{+}$ cells (Figure 9A). These cells were highly CD24+ (Figure 9A), were distinct from $\mathrm{CD} 14^{+} \mathrm{BM}$ monocytes, and displayed phenotypic characteristics of $\mathrm{cDC1}$ (data not shown). When stimulated with the mAbs to human CD24 ( $\alpha$ hCD24 mAb), these BM cDC1s upregulated cell-surface expression of mSCF (Figure 9B) similarly to what was observed for their murine splenic $\mathrm{CDC} 1$ DC counterparts (Figure 7).

CD24 expression was also detected on BM cells with the phenotypic properties of neutrophils and B lymphocytes (data not shown), which was similar to what occurred in their murine counterparts. Importantly, when the resident CD24 ${ }^{\text {hi }}$ cells, i.e., B cells, neutrophils, $\mathrm{CD}_{14}{ }^{+}$cells, and $\mathrm{cDC} 1$ s within $\mathrm{BM}$ aspirates, were sorted and stimulated in vitro with control IgG or ahCD24 Abs, the
$\mathrm{CD} 24^{\text {hi }} \mathrm{cDC} 1 \mathrm{~s}$ displayed the highest basal mSCF gene expression and this cell type was most sensitive to $\mathrm{mSCF}$ (and SSCF) upregulation following $\alpha$ hCD24 Ab treatment (Figure 9, C and D).

To further explore the impact of $\mathrm{CD} 24$ in regulating erythropoiesis by human erythroid progenitor cells in vivo, we analyzed the effect of administration of mouse $\alpha \mathrm{hCD} 24 \mathrm{Abs}$ on the response of humanized mice. These humanized mice (hNSG, where NSG indicates NOD/ SCID/IL-2R $\gamma$-null) were engrafted with hematopoietic cells (CD45 ${ }^{+}$) of human origin with various efficiencies (Figure 10A). Furthermore, the $\mathrm{CD} 141^{+} \mathrm{DC}$ subset $\left(\mathrm{hCD} 141^{+} \mathrm{mCD} 11 \mathrm{c}^{-}\right.$) was detected along with murine DCs (hCD141 ${ }^{-} \mathrm{mCD} 11 \mathrm{c}^{+}$) (Figure 10B) within the spleen. Control nonhumanized (NSG) chimeric mice receiving mAbs to mouse CD24 exhibited the expected expansion of splenic CD45- ${ }^{-}{ }^{-119}{ }^{+}$, predominantly $\mathrm{CD}^{+} 1^{+}$, erythroid progenitors of mouse origin (Figure 10, C and D) as well as transient reticulocytosis (Figure 10E). On the other hand, administration of mouse mAbs to human CD24 to NSG mice had a minimal effect on these markers of induced erythropoiesis, i.e., comparable to that of control IgG (Figure 10, C-E).

In the case of hNSG chimeric mice, administration of mAbs to either the mouse or the human CD24 had a modest but comparable effect on splenic CD $45^{-}$Ter $119^{+}$cell frequencies above the background observed with control mAbs (Figure 10D) as well as a comparable induction of reticulocytosis (Figure 10E), which again was above the background observed with control IgG-treated hNSG chimeric mice. 
A

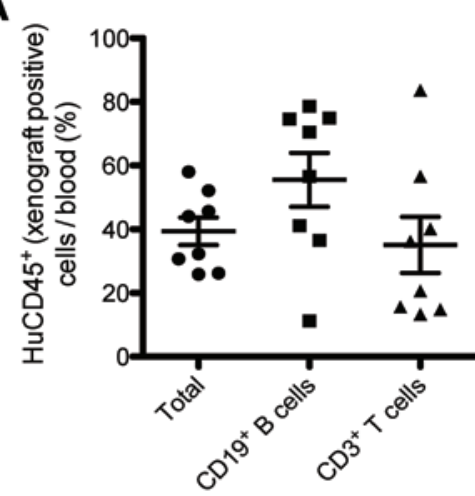

D

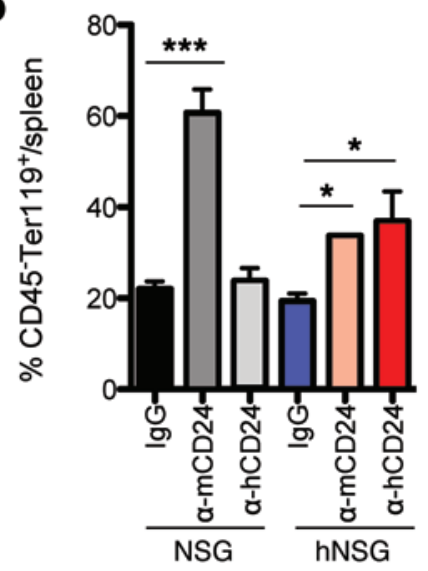

B

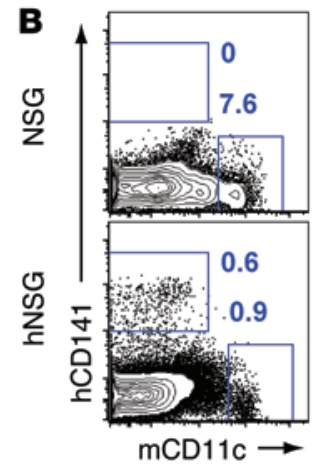

E

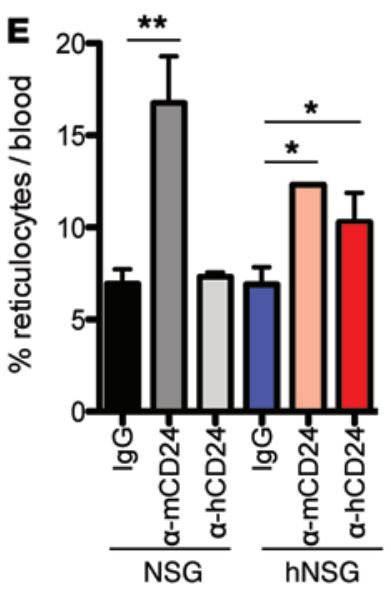

C

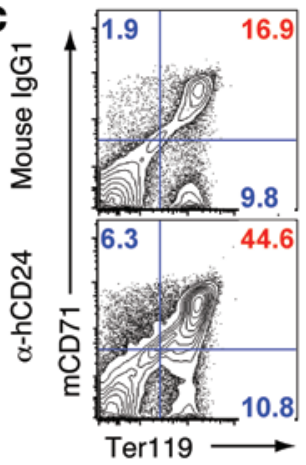

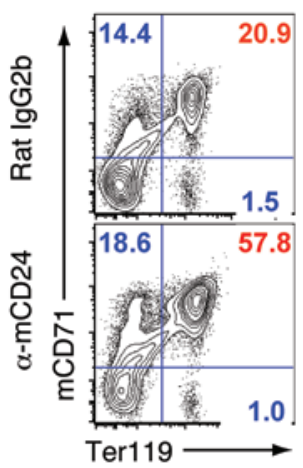

Figure 10. In vivo erythropoietic responses to human CD24 engagement. (A and B) NSC and hNSC mice were examined for the efficiency of engraftment of human origin lymphocytes in blood (A) and DCs (anti-human CD141 for human DCs and antimouse CD11c for murine DCs) in the spleen (B). Humanized mice were treated i.p. with control Igs, Abs against mouse (M1/69), or human anti-CD24 Abs (eBioSN3 or ML5). Ab-treated mice were necropsied at day 5 in order to identify murine erythroid progenitors in the spleens after costaining with a cocktail of Abs against murine CD71, Ter119, and CD45 (C and D) and the percentage of circulating mouse-origin reticulocytes in the blood (E) $(n=5-8)$. Data represent mean \pm SEM; ${ }^{*} P<0.05$; ${ }^{* *} P<0.01$; ${ }^{* *} P<0.001$ (2-tailed, unpaired Student's $t$ test).

We also observed in the hNSG treated with a human CD24 that the erythroid progenitors detected in the spleens were predominantly of murine origin (Figure 10C). Our failure to detect expansion of these terminal erythroid elements of human origin (Supplemental Figure 10) most likely reflects the phagocytosis of later-stage human erythroid progenitors by murine macrophages (32). Nevertheless, this demonstration of induction of erythropoiesis by engagement of the human CD24 in vivo suggests that this molecule may serve as a sensor of stress-triggering erythropoiesis in the human. In this connection, it is noteworthy that murine EPO production was stimulated by administration of mAbs to human CD24 to hNSG but at a level approximately one-third that of the EPO induced in these animals by treatment with mAbs to mouse CD24 (data not shown).

\section{Discussion}

In this report, we demonstrate a previously unappreciated role for DCs and, most prominently, the $\mathrm{cDC} 1\left(\mathrm{CD} 8 \alpha^{+} ; \mathrm{XCR}^{+} \mathrm{CLEC} 9 \mathrm{~A}^{+}\right)$ DC subset (15-17), in regulating the initiation of stress-induced erythropoiesis. We find that engagement of the molecular stress sensor CD24 on the DCs results in the rapid upregulation of SCF expression on the DCs. This in turn triggers a Kit-dependent activation and proliferative response of early erythroid progenitors and subsequent EPO-dependent erythroblast expansion and reticulocytosis. Furthermore, we demonstrate that the cDC1-dependent, stress sensor engagement-mediated erythropoiesis response, in part at least, accounts for the erythropoietic stimulus resulting from pathologic stress triggered by hemorrhage, hypoxia, and BM suppression. Thus, our analysis has revealed $\mathrm{cDC} 1 \mathrm{~s}$ as regulators of stress erythropoiesis, likely acting through recognition of stresses (e.g., DAMPs) recognized by one or more cell-associated stress sensors. Of note, our analysis further suggests that this contribution of DCs to the initiation of stress erythropoiesis is distinct from the role of macrophages operating downstream in this process.

Cells of the MPS have long been appreciated as serving an essential role in both basal and stress-associated erythropoiesis (1). In particular, recent evidence suggests that $\mathrm{CD} 169^{+}$macrophages may carry out this critical role by supporting late-stage erythroid differentiation/ maturation and possibly regulating iron metabolism $(3,14)$. Likewise, the stem cell growth factor receptor Kit is an essential regulator of hematopoiesis, including erythropoiesis $(28,33)$, but a link between Kit expression and the requirement for cells of the MPS in the early induction of stress erythropoiesis has, to date, not been clearly established. The results herein establish one such link through upregulation of expression of the Kit ligand SCF on DCs, most prominently (but not exclusively) the $\mathrm{CDC1}$ subset following engagement of a particular stress sensor, i.e., CD24. Although the murine spleen $\mathrm{CDC1}\left(\mathrm{CD} 8 \alpha^{+}\right.$ DC) subset is believed to be localized predominantly in the white pulp T cell zones, a fraction of the splenic CD $8 \alpha^{+}$DCs localize in the white pulp marginal zone (25) and possibly the red pulp (24), where they are poised to sense danger signals present in the circulation, e.g., alarmins and other self constituents released from damaged or stressed cells (25), and thereby have the potential to initiate stress erythropoiesis. In this connection, it will be important to determine the anatomical localization of these stress-sensing DCs and their geographic (anatomical) relationship to the $\mathrm{CD} 169^{+}$macrophages residing within the erythroblast islands found in the BM and spleen $(14,34)$. 
CD24 was initially identified as a costimulatory ligand supporting the induction of adaptive immune responses $(35,36)$. More recently, along with its costimulatory role in supporting naive $\mathrm{CD}^{+} \mathrm{T}$ cell induction $(18,37), \mathrm{CD} 24$ has been demonstrated as serving as a stress sensor recognizing DAMPs, i.e., molecules released from inflamed and dying cells (31). The mechanism of signal transduction by this glycophosphatidylinositol-linked (GPI-linked) glycoprotein is not fully understood, and transduction of either activating or inhibitory signals has been reported following CD24 engagement $(31,38,39)$. The results reported here suggest that when expressed (at a high level) on at least one subset of DCs, i.e., cDC1s, engagement of CD24 triggers the upregulated expression of SCF by this cell type with consequent effects on stress erythropoiesis. In this context, it is important to emphasize that a deficiency in CD24 does not affect basal/homeostatic erythropoiesis and only diminishes (or more precisely delays) pathologic stress-induced erythropoiesis. Thus, it is likely that CD24 is only one of several, e.g., herpesvirus entry mediator (HVEM) (40) and acetate (41), stress sensors capable of regulating erythropoiesis in response to stress.

In vivo "neutralization" of one CD24 ligand, HMGB1, by Abs during stress induction did partially blunt (delay) the onset of the erythropoietic response. HMGB1 likely represents only one of a variety of alarmins (DAMPs) capable of engaging this and other stress sensors. Our inability to reproducibly stimulate erythropoesis following a single injection of recombinant HMGB1 may reflect the need for this molecule to fold in an optimal conformation or to associate with a particular coligand, e.g., nucleic acid, for maximum potency (42). The relative contribution of various stress sensors and their ligands to the control of stress erythropoiesis in both the human and the mouse model will need to be determined by additional experimentation.

The selective deficiency in the CD $8 \alpha^{+}$DC lineage in Baft3 $3^{-1-}$ mice markedly diminishes erythropoiesis stimulated by CD24 engagement in vivo. However, as noted above for $\mathrm{Cd} 24^{-/-}$mice, this DC subset deficiency does not affect basal erythropoiesis or completely abolish rbc formation in response to stress. Consequently, other cell types, most notably other subsets of CD11 $\mathrm{c}^{+}$ cDC, e.g., cDC2, likely contribute to the regulation of stress erythropoiesis. However, the $\mathrm{CD} 8 \alpha^{+} \mathrm{DC}$ subset from the murine spleen along with its XCR $1^{+} \mathrm{CLEC} 9{ }^{+} \mathrm{DC}$ counterpart from the human $\mathrm{BM}$ upregulate the critical Kit ligand SCF following CD24 engagement and do so more effectively than other $\mathrm{CD} 24^{+}$cell types, including other DC subsets as well as B lymphocytes and neutrophils that express CD24 at elevated levels in the human as well.

CD24 is a relatively ubiquitous cell surface glycoprotein displayed at varying levels on many cell types. The finding that acute transient CD24 engagement in vivo selectively enhances stress erythropoiesis with minimal impact on other cellular processes, e.g., expansion of other hematopoietic cells, suggests that the consequence of CD24 signaling, i.e., transduction of a positive or negative signal and selective upregulation of SCF in certain cell types, may reflect both the level of CD24 expression on the cell and the cell type in which CD24 is engaged. BMP4 has also been implicated in the regulation of erythropoiesis $(10,43,44)$. However, in this model, we observed a modest effect of CD24 engagement on BMP 4 expression by CD $8 \alpha^{+}$DCs, at least in vitro (T.S. Kim, unpub- lished observations). The interplay among BMP4, SCF, and factors such as HIF-1/2 $\alpha$ as well as the cell types expressing these factors in mediating the control of stress erythropoiesis (33) awaits additional experimentation.

Our findings in this model of stress erythropoiesis induction suggest that following stress, there is early activation and expansion of early erythroid progenitors whose expansion is dependent on Kit signaling by the ligand SCF expressed on one or more distinct subsets of $\mathrm{cDC}$, most prominently cDC1s. This DC-orchestrated initial Kit signaling event is transient and is complemented by subsequent EPO-dependent signaling events (as well as continued DC-independent Kit signaling; T.S. Kim, unpublished observations) resulting in terminal erythrocyte differentiation.

We observed that in vivo engagement of CD24 also stimulated EPO production and release into the circulation. This was accompanied by the upregulation in the kidneys of $\mathrm{O}_{2}$-sensitive HIF transcription factors, notably HIF- $2 \alpha$, which plays a central role in the regulation of EPO expression and, consequently, erythropoiesis (45). While EPO production in response to CD24 engagement was independent of the $\mathrm{SCF} / \mathrm{Kit}$ interaction necessary for early erythroid progenitor expansion, we provide evidence to suggest that the production of EPO by the murine kidney is dependent on engagement of CD24 on a CD11 $\mathrm{c}^{+}$cell type, most likely, a kidney DC population. Additional studies will need to be carried out to characterize the cell type(s) and the signal transduction events associated with CD24-stimulated EPO production in the kidney.

Cells of the immune system respond to stress. In this report, we demonstrate and analyze what we believe to be a novel function of one immune cell type, that is, the DCs, in the control of rbc production in response to stress. Our findings provide a framework for a detailed analysis of the regulation of stress (and potentially homeostatic) erythropoiesis in vitro and in vivo. Perhaps more importantly, they open up the possibility of new therapeutic targets for the treatment of anemia to replace or supplement treatments based on erythropoiesis-stimulating agents (ESAs) and iron metabolism.

\section{Methods}

Mice and humanized mice. Female C57BL/6 (B6, H-2 $)$ and Balb/c $\left(\mathrm{H}-2^{\mathrm{d}}\right)$ mice ranging from 8 to 12 weeks old were purchased from the National Cancer Institute (Frederick, Maryland, USA). Batf3-/- mice on a 129S6/ SvEv background were previously generated (23) and were backcrossed at least 10 generations to the C57BL/6 background prior to use in our experiments (18). Transgenic ( $\mathrm{Tg}$ ) mice expressing nonhuman primate DTR under the control of the murine Cd11c promoter (Cd11c-DTR; ref. 20) were purchased from the Jackson Laboratory and bred in house. To deplete CD11c cells, DTR Tg mice were injected i.p. with DTx (100 $\mathrm{ng} / 250$ ul in PBS, Sigma-Aldrich) unless otherwise stated. We obtained female double-heterozygous Kit-mutant mice $\left(\mathrm{Kit}^{W} / \mathrm{Kit}^{W-v}\right)$ on a WBB6 F1 hybrid background (Jackson Laboratory, stock no. 100410) as well as WT sex- and age-matched controls from the same colony and housed mice under standard conditions. Rag1/-, NSG, and hNSG mice were purchased from the Jackson Laboratory. The hNSG mice reconstituted with human $\mathrm{CD} 34^{+}$cells were prepared by the Jackson Laboratory and were analyzed 7 to 8 weeks after reconstitution. Cd24-null mice were a gift from Dan Theodorescu (University of Colorado, Boulder, Colorado, USA). All animals were housed in specific pathogen-free facilities at the University of Virginia School of Medicine animal facility. 
Colony-forming unit assays. Spleen cell suspensions for colonyforming assays were prepared in RPMI-1640 media supplemented with $10 \% \mathrm{FBS}$ and antibiotics at $\times 10$ concentrations. rbc from spleen cell suspensions were lysed in rbc Lysing buffer prior to plating. Mature BFU-E and CFU-E were quantified in spleen cell suspensions using MethoCult SF M3436 medium. Briefly, $1 \times 10^{6}$ cells $/ \mathrm{ml}$ or $2 \times 10^{5}$ cells/ $\mathrm{ml}$ for spleen were prepared in RPMI 1640 and $0.3 \mathrm{ml}$ was added to 3 $\mathrm{ml}$ of MethoCult medium for triplicate cultures. The mix was vortexed, allowed to stand for 5 minutes, and dispensed into 35-mm culture dishes (StemCell Technologies) using a 16-gauge blunt-end needle and a 3-cc syringe, $1.1 \mathrm{ml}$ per dish. The cultures were incubated at $37^{\circ} \mathrm{C}, 5 \%$ $\mathrm{CO}_{2}$ in air and at $95 \%$ or more humidity. CFU-E and BFU-E were identified and counted at 2 and 7 (or day 10) days in culture, respectively.

Macrophage depletion in vivo. Macrophages were depleted in vivo with i.v. infusion of PBS-coated liposomes (inactive forms) as a control or clodronate-encapsulated liposomes (active forms) (200 $\mu \mathrm{l} /$ injection). Preassembled liposomes containing dichloromethylene diphosphonate $\left(\mathrm{Cl}_{2} \mathrm{MDP}\right.$, also called clodronate) or PBS were purchased from Encapsula NanoSciences LLC and used according to the manufacturer's suggestions.

Single-cell preparation. Single-cell suspensions from spleens and $\mathrm{BM}$ were prepared by pushing samples through a $70-\mu \mathrm{m}$ cell strainer (3) without enzymatic digestion. The rbc in the cell suspension were lysed using ammonium chloride buffer. Cells were washed and resuspended in FACS buffer containing $1 \times$ PBS, 2\% FBS, 10 mM EDTA, and $0.01 \% \mathrm{NaN}_{3}$ for Ab staining or MACS buffer containing $1 \times \mathrm{PBS}, 2 \%$ FBS, and $10 \mathrm{mM}$ EDTA for sorting. Viable nucleated cells in the suspensions were counted using a hemacytometer following exclusion of dead cells using Trypan blue dye. Unprocessed fresh human BM aspirates were purchased from Lonza and were used after rbc lysis.

$H b, H C T$, and reticulocyte measurements. To obtain hematological values, retroorbital blood samples $(30-50 \mu \mathrm{l})$ were collected in $\mathrm{K}_{2}$ EDTA-treated microtainer and analyzed on the Hemavet $850 \mathrm{FS}$ automated CBC analyzer (Drew Scientific) within 2 hours of blood collection. The percentage of reticulocytes in blood was assessed by flow cytometry using thiazole orange stain as previously described (46). Blood $(5 \mu \mathrm{l})$ was placed in $1 \mathrm{ml}$ of thiazole orange solution and was incubated at least 1 hour at room temperature before flow cytometric analyses.

Hypoxia exposure, cisplatin, phlebotomy, imatinib, and HMGB1 treatment. Hypoxia was induced by purging the hypoxia chamber (provided by Lisa Palmer, University of Virginia) with nitrogen to an approximately $10 \% \mathrm{O}_{2}$ atmosphere. WT and $\mathrm{KO}$ mice calibrated under normoxia (room air, 21\% oxygen) were cohoused under hypoxic conditions for the indicated periods. To induce cisplatininduced anemia, mice were injected i.v. with $3 \mathrm{mg} / \mathrm{kg}$ cisplatin (cisDiammineplatinum(II) dichloride, Sigma-Aldrich) dissolved in $\mathrm{dd}_{2} \mathrm{O}$ daily from day 0 to day 2 . Phlebotomy was performed by retroorbital puncture $(400 \mu \mathrm{l})$ at each time point for 3 consecutive days. To inhibit Kit signaling in vivo, mice were treated i.p. with imatinib mesylate (Gleevec; Novartis) dissolved in $0.9 \% \mathrm{NaCl}$ daily at $5 \mathrm{mg} / \mathrm{kg}$ from day 0 to day 2. For HMGB1 treatment in vitro, recombinant human HMGB1 (Sigma-Aldrich, $20 \mu \mathrm{g} / \mathrm{ml}$ ) was incubated in complete RPMI culture media for DC stimulation.

BM transplantation. Mice were irradiated $(1,150 \mathrm{~Gy})$ in a Cesium Mark 1 irradiator (JL Shepherd and Associates). Then $2 \times 10^{6}$ rbc-lysed $\mathrm{BM}$ nucleated cells were injected i.v. within 1 hour of the final irra- diation. Mice were allowed to recover from BM transfer for at least 6 weeks before being used for further experiments.

Splenectomy. Mice were anesthetized i.p. by injection of tribromoethanol (Sigma-Aldrich). To remove spleen, a transverse incision was made on the left lower back to expose the spleen. The gastrosplenic ligament was cut, the splenic blood vessels were tied off with silk suture, and the spleen was removed. The incisions were closed with sutures on the body wall followed by wound clips on the skin. The splenectomized mice were allowed to recover for at least 2 weeks before further experiments.

Serum EPO. Serum EPO concentrations were determined by ELISA using the kit from R\&D Biosystems according to the instructions provided.

DC differentiation in vitro. Human BMDCs were generated by culturing total $\mathrm{BM}$ aspirates (Lonza) after rbc lysis at $2 \times 10^{6}$ cells $/ \mathrm{ml}$ in complete RPMI medium. Cell culture was performed in polystyrene 6- or 24-well plates (Falcon, BD) with $3 \mathrm{ml}$ or $0.75 \mathrm{ml}$ of cell suspension per well, respectively. Fresh complete RPMI medium supplemented with $1000 \mathrm{U} / \mathrm{ml} \mathrm{rHu} \mathrm{IL-4} \mathrm{(Promocell),} 800 \mathrm{U} / \mathrm{ml} \mathrm{rHu} \mathrm{GM-CSF}$ (Leucomax, Novartis), and $200 \mathrm{U} / \mathrm{ml} \mathrm{rHu} \mathrm{Flt3L}$ (Promocell) preheated at $37^{\circ} \mathrm{C}$ was added. At days 3 and 6 , half the culture medium was replaced by fresh preheated complete RPMI medium with $500 \mathrm{U} / \mathrm{ml} \mathrm{rHu} \mathrm{IL-4,}$ $800 \mathrm{U} / \mathrm{ml} \mathrm{rHu} \mathrm{GM-CSF}$, and $200 \mathrm{U} / \mathrm{ml} \mathrm{rHu} \mathrm{Flt3L.} \mathrm{Nonadherent}$ immature DCs (iDCs) harvested at day 7 were stimulated with Abs. Murine BMDCs were cultured with complete RPMI medium with 40 $\mathrm{U} / \mathrm{ml}$ mouse GM-CSF and $100 \mathrm{U} / \mathrm{ml}$ murine Flt3L.

Abs, flow cytometry, and cell cycle analyses. Fluorochrome-conjugated or biotinylated monoclonal Abs specific to mouse Ly6G (clone 1A8), Gr-1 (clone RB6-8C5), CD115 (clone AFS98), B220 (clone RA3-6B2), CD11b (clone M1/70), CD45 (clone 30-F11), CD45.1 (clone A20), CD45.2 (clone 104), Ter119 (clone TER-119), CD71 (clone R17217), CD24 (clone M1/69), CD11c (clone HL3), CD16/CD32 (clone 93), MHC class II (clone M5/114), CD168 (clone 3D6.112), CD8 $\alpha$, isotype control Abs, and the secondary reagents were purchased from BD Biosciences. Abs to F4/80 (CI:A3.1) were purchased from AbD Serotec. Rabbit polyclonal Abs to murine SCF were purchased from Abcam. Anti-human Abs, HLA-DR (L243), BDCA-3/CD141 (AD5-14H12), CD11c (B-ly6), CD14 (M/P9), CD20 (L27), CD56 (B159), CD3 (OKT3), CD11b (ICRF44), CD71 (M-A712), CD235a (GA-R2), CLEC9A (3A4/CLEC9A), and isotype-matched control Abs were purchased from BD Biosciences. Polyclonal Abs against human XCR1 were from R\&D Systems. Anti-human CD24 Abs were purchased from either Biolegend (ML5) or eBiosciences (eBioSN3). Rabbit polyclonal Abs to human SCF were purchased from Abcam. For tissue culture stimulation, anti-human CD24 Abs were dialyzed 5 times in PBS to remove any residual sodium azide in house. Human and mouse cells were preincubated on ice with mouse serum (Jackson ImmunoResearch Laboratories) plus purified IgG2a (BD Biosciences), or anti-mouse CD16/CD32 (93; eBiosciences), respectively, to block Fc receptors and then stained with appropriate $\mathrm{Ab}$ combinations.

For cell cycle analysis, we used the APC-BrdU Flow Kit (BD Biosciences - Pharmingen) according to the manufacturer's instructions. In brief, $1 \mathrm{mg}$ of BrdU was administered to mice by i.p. injection, and the BM and spleen were harvested 1 hour after BrdU administration as described in the manual. Mice that were not injected with BrdU were used as a control. For nuclear staining of Ki-67, cell suspensions were 
stained for surface for 30 minutes on ice, followed by use of a FOXP3staining kit according to the manufacturer's instructions (eBiosciences). Samples were run in a FACSCanto II system equipped with 3 lasers. Analysis was performed using FlowJo software (Tree Star Inc.).

In vivo blockade by $A b$ administration. To block receptor/ligand interactions in vivo, mice were treated i.p. with $200 \mu \mathrm{g}$ of anti-EPO (MAB959, R\&D Systems), anti-HMGB1 (3E8, BioLegend), or anti-Kit Abs (ACK2, BD), respectively.

RNA isolation and quantitative real-time PCR. Total RNA was extracted from tissues using TRIzol (Invitrogen) according to the manufacturer's instructions. cDNA was prepared with SuperScript II Reverse Transcriptase (Invitrogen) from $2 \mu \mathrm{g}$ total RNA using random hexamers. Real-time PCR was performed in an ABI PRISM Cycler (Life Technologies) with a SYBR Green PCR Kit from Applied Biosystems (Life Technologies). The PCR protocol consisted of 1 cycle at $95^{\circ} \mathrm{C}$ (10 minutes) followed by 40 cycles of $95^{\circ} \mathrm{C}$ ( 15 seconds) and $60^{\circ} \mathrm{C}$ ( 1 minute). Expression levels of evaluated genes were calculated with the comparative $\Delta \mathrm{Ct}$ method, and relative abundance was quantified with the $2^{-\Delta \mathrm{Ct}}$ method. Expression of hypoxanthine-guanine phosphoribosyltransferase ( $H p r t)$ was used as a standard. The average threshold cycle number (CRtR) for each tested mRNA was used to quantify the relative expression of each gene. The primers used for qRT-PCR are summarized in Supplemental Table 1.

Statistics. Unless otherwise indicated in the figure legends, unpaired Student's $t$ test was used in all analyses. Statistical analyses were performed with Prism 5.0c software (GraphPad Software). Data in the bar graphs are shown as mean \pm SEM. $P$ values of less than 0.05 were considered statistically significant.
Study approval. All mouse experiments were carried out according to the NIH's Guide for the Care and Use of Laboratory Animals (8th ed. The National Academies Press. 2011), and animal care and experimental procedures were approved by the University of Virginia Animal Care and Use Committee.

\section{Acknowledgments}

We thank Barbara Small and Sowmya Narayanan for excellent technical assistance and members of the Braciale laboratory for insightful comments and helpful discussions. We also would like to thank A. Goldfarb, L. Delehanty, K. Tung, H. Qiao, L. Palmer, and V. Laubach (University of Virginia); K. Tracey and H. Yang (The Feinstein Institute for Medical Research); Dan Theodorescu (University of Colorado); and K. Murphy (Washington University) for reagents, mice, technical support, and advice. This study is supported by the NIH (R01 AI-15608, U19 AI-83024, and R01 HL - 33391 to T.J. Braciale), the Ivy Foundation (to T.J. Braciale and T.S. Kim), and the Virginia Innovation Partnership - i6 Challenge (to T.J. Braciale and T.S. Kim).

Address correspondence to: Thomas J. Braciale, Carter Immunology Center, University of Virginia, P.O. Box 801386, Charlottesville, Virginia 22908, USA. Phone: 434.924.1219; E-mail: tjb2r@ virginia.edu. Or to: Taeg S. Kim, Carter Immunology Center, University of Virginia, P.O. Box 801386, Charlottesville, Viriginia 22908, USA. Phone: 434.924.1277; E-mail: tsk5g@virginia.edu.

Paul C. Trampont's present address is: Department of Hematology/Cytometry, Covance Inc., Indianapolis, Indiana, USA.
1. Bessis M. [Erythroblastic island, functional unity of bone marrow]. Rev Hematol. 1958;13(1):8-11.

2. Chasis JA, Mohandas N. Erythroblastic islands: niches for erythropoiesis. Blood. 2008;112(3):470-478.

3. Ramos P, et al. Macrophages support pathological erythropoiesis in polycythemia vera and betathalassemia. Nat Med. 2013;19(4):437-445.

4. Wu H, Klingmuller U, Besmer P, Lodish HF. Interaction of the erythropoietin and stem-cell-factor receptors. Nature. 1995;377(6546):242-246.

5. Hattangadi SM, Wong P, Zhang L, Flygare J, Lodish HF. From stem cell to red cell: regulation of erythropoiesis at multiple levels by multiple proteins, RNAs, and chromatin modifications. Blood. 2011;118(24):6258-6268.

6. Socolovsky M. Molecular insights into stress erythropoiesis. Curr Opin Hematol. 2007;14(3):215-224.

7. de Back DZ, Kostova EB, van Kraaij M, van den Berg TK, van Bruggen R. Of macrophages and red blood cells; a complex love story. Front Physiol. 2014;5:9.

8. Bunn HF, Poyton RO. Oxygen sensing and molecular adaptation to hypoxia. Physiol Rev. 1996;76(3):839-885.

9. Semenza GL. Involvement of oxygen-sensing pathways in physiologic and pathologic erythropoiesis. Blood. 2009;114(10):2015-2019.

10. Paulson RF, Shi L, Wu DC. Stress erythropoiesis: new signals and new stress progenitor cells. Curr Opin Hematol. 2011;18(3):139-145.

11. Lenox LE, Shi L, Hegde S, Paulson RF. Extramed- ullary erythropoiesis in the adult liver requires BMP-4/Smad5-dependent signaling. Exp Hematol. 2009;37(5):549-558.

12. Ploemacher RE, van Soest PL. Morphological investigation on phenylhydrazine-induced erythropoiesis in the adult mouse liver. Cell Tissue Res. 1977;178(4):435-461.

13. Perry JM, Harandi OF, Porayette P, Hegde S, Kannan AK, Paulson RF. Maintenance of the BMP4dependent stress erythropoiesis pathway in the murine spleen requires hedgehog signaling. Blood. 2009;113(4):911-918.

14. Chow A, et al. CD169(+) macrophages provide a niche promoting erythropoiesis under homeostasis and stress. Nat Med. 2013;19(4):429-436.

15. Shortman K, Heath WR. The CD8 ${ }^{+}$dendritic cell subset. Immunol Rev. 2010;234(1):18-31.

16. Jongbloed SL, et al. Human CD $141^{+}$(BDCA-3)+ dendritic cells (DCs) represent a unique myeloid DC subset that cross-presents necrotic cell antigens. JExp Med. 2010;207(6):1247-1260.

17. Bachem A, et al. Superior antigen crosspresentation and XCR1 expression define human $\mathrm{CD} 11 \mathrm{c}^{+} \mathrm{CD} 141^{+}$cells as homologues of mouse CD8 ${ }^{+}$dendritic cells. J Exp Med. 2010;207(6):1273-1281.

18. Kim TS, Gorski SA, Hahn S, Murphy KM, Braciale TJ. Distinct dendritic cell subsets dictate the fate decision between effector and memory CD8(+) $\mathrm{T}$ cell differentiation by a CD24-dependent mechanism. Immunity. 2014;40(3):400-413.

19. Socolovsky M, Nam H, Fleming MD, Haase $\mathrm{VH}$, Brugnara C, Lodish HF. Ineffective eryth- ropoiesis in Stat5a(-/-)5b(-/-) mice due to decreased survival of early erythroblasts. Blood. 2001;98(12):3261-3273.

20. Jung S, et al. In vivo depletion of CD11c(+) dendritic cells abrogates priming of CD8(+) T cells by exogenous cell-associated antigens. Immunity. 2002;17(2):211-220.

21. Guilliams M, et al. Dendritic cells, monocytes and macrophages: a unified nomenclature based on ontogeny. Nat Rev Immunol. 2014;14(8):571-578.

22. Hashimoto D, Miller J, Merad M. Dendritic cell and macrophage heterogeneity in vivo. Immunity. 2011;35(3):323-335.

23. Hildner $\mathrm{K}$, et al. Batf3 deficiency reveals a critical role for $\mathrm{CD} 8 \alpha^{+}$dendritic cells in cytotoxic T cell immunity. Science. 2008;322(5904):1097-1100.

24. Kool M, et al. The ubiquitin-editing protein A20 prevents dendritic cell activation, recognition of apoptotic cells, and systemic autoimmunity. Immunity. 2011;35(1):82-96.

25. Qiu CH, Miyake Y, Kaise H, Kitamura H, Ohara O, Tanaka M. Novel subset of CD8\{alpha\} dendritic cells localized in the marginal zone is responsible for tolerance to cell-associated antigens. JImmunol. 2009;182(7):4127-4136.

26. Miyake Y, Asano K, Kaise H, Uemura M, Nakayama M, Tanaka M. Critical role of macrophages in the marginal zone in the suppression of immune responses to apoptotic cell-associated antigens. J Clin Invest. 2007;117(8):2268-2278.

27. Broudy VC, Lin NL, Priestley GV, Nocka K, Wolf NS. Interaction of stem cell factor and its receptor c-kit mediates lodgment and acute expansion 
of hematopoietic cells in the murine spleen. Blood.1996;88(1):75-81.

28. Broudy VC. Stem cell factor and hematopoiesis. Blood.1997;90(4):1345-1364.

29. Koury MJ. Erythropoietin: the story of hypoxia and a finely regulated hematopoietic hormone. Exp Hematol. 2005;33(11):1263-1270.

30. Liu Y, Pop R, Sadegh C, Brugnara C, Haase VH, Socolovsky M. Suppression of Fas-FasL coexpression by erythropoietin mediates erythroblast expansion during the erythropoietic stress response in vivo. Blood. 2006;108(1):123-133.

31. Chen GY, Tang J, Zheng P, Liu Y. CD24 and Siglec-10 selectively repress tissue damage-induced immune responses. Science. 2009;323(5922):1722-1725.

32. Hu Z, Van Rooijen N, Yang YG. Macrophages prevent human red blood cell reconstitution in immunodeficient mice. Blood. 2011;118(22):5938-5946.

33. Perry JM, Harandi OF, Paulson RF. BMP4, SCF, and hypoxia cooperatively regulate the expansion of murine stress erythroid progenitors.
Blood. 2007;109(10):4494-4502.

34. Chow A, et al. Bone marrow $\mathrm{CD} 169^{+}$macrophages promote the retention of hematopoietic stem and progenitor cells in the mesenchymal stem cell niche. J Exp Med. 2011;208(2):261-271.

35. Liu Y, Wenger RH, Zhao M, Nielsen PJ. Distinct costimulatory molecules are required for the induction of effector and memory cytotoxic $\mathrm{T}$ lymphocytes. JExp Med.1997;185(2):251-262.

36. Liu Y, Jones B, Aruffo A, Sullivan KM, Linsley PS, Janeway CA Jr. Heat-stable antigen is a costimulatory molecule for CD $4 \mathrm{~T}$ cell growth. J Exp Med. 1992;175(2):437-445.

37. Li O, Zheng P, Liu Y. CD24 expression on T cells is required for optimal $\mathrm{T}$ cell proliferation in lymphopenic host. JExp Med. 2004;200(8):1083-1089.

38. Hubbe M, Altevogt P. Heat-stable antigen/CD24 on mouse T lymphocytes: evidence for a costimulatory function. Eur J Immunol. 1994;24(3):731-737.

39. Wu Y, Zhou Q, Zheng P, Liu Y. CD28-independent induction of Thelper cells and immunoglobulin class switches requires costimulation by the heatstable antigen. J Exp Med.1998;187(7):1151-1156.
40. Sakoda Y, et al. Herpesvirus entry mediator regulates hypoxia-inducible factor-1 $\alpha$ and erythropoiesis in mice. JClin Invest. 2011;121(12):4810-4819.

41. Xu M, et al. An acetate switch regulates stress erythropoiesis. Nat Med. 2014;20(9):1018-1026.

42. Andersson U, Tracey KJ. HMGB1 is a therapeutic target for sterile inflammation and infection. Annu Rev Immunol. 2011;29:139-162.

43. Harandi OF, Hedge S, Wu DC, McKeone D, Paulson RF. Murine erythroid short-term radioprotection requires a BMP4-dependent, self-renewing population of stress erythroid progenitors. J Clin Invest. 2010;120(12):4507-4519.

44. Lenox LE, Perry JM, Paulson RF. BMP4 and Madh5 regulate the erythroid response to acute anemia. Blood. 2005;105(7):2741-2748.

45. Gruber M, Hu CJ, Johnson RS, Brown EJ, Keith B, Simon MC. Acute postnatal ablation of Hif$2 \alpha$ results in anemia. Proc Natl Acad Sci U S A. 2007;104(7):2301-2306.

46. Lee LG, Chen CH, Chiu LA. Thiazole orange: a new dye for reticulocyte analysis. Cytometry. 1986;7(6):508-517. 